\title{
The nonuniqueness of the tangent cones at infinity of Ricci-flat manifolds
}

\author{
Kota Hattori \\ Keio University \\ 3-14-1 Hiyoshi, Kohoku, Yokohama 223-8522, Japan \\ hattori@math.keio.ac.jp
}

\begin{abstract}
It is shown by Colding and Minicozzi the uniqueness of the tangent cones at infinity of Ricci-flat manifolds with Euclidean volume growth which has at least one tangent cone at infinity with a smooth cross section. In this paper we raise an example of the Ricci-flat manifold implying that the assumption for the volume growth in the above result is essential. More precisely, we construct a complete Ricci-flat manifold of dimension 4 with non-Euclidean volume growth who has infinitely many tangent cones at infinity and one of them has a smooth cross section.
\end{abstract}

\section{Introduction}

For a complete Riemannian manifold $(X, g)$ with nonnegative Ricci curvature, it is shown by Gromov's Compactness Theorem that if one take a sequence

$$
a_{1}>a_{2}>\cdots>a_{i}>\cdots>0
$$

such that $\lim _{i \rightarrow \infty} a_{i}=0$, then there is a subsequence $\left\{a_{i(j)}\right\}_{j}$ such that $\left(X, a_{i(j)} g, p\right)$ converges to a pointed metric space $(Y, d, q)$ as $j \rightarrow \infty$ in the sense of the pointed Gromov-Hausdorff topology [9] [10]. The limit $(Y, d, q)$ is called the tangent cone at infinity of $(X, g)$. In general, the pointed GromovHausdorff limit might depend on the choice of $\left\{a_{i}\right\}_{i}$ or its subsequences.

The tangent cone at infinity is said to be unique if the isometry classes of the limits are independent of the choice of $\left\{a_{i}\right\}$ and its subsequences, and Colding and Minicozzi showed the next uniqueness theorem under the certain assumptions. 
Theorem $1.1([6])$. Let $(X, g)$ be a Ricci-flat manifold with Euclidean volume growth, and suppose that one of the tangent cone at infinity has a smooth cross section. Then the tangent cone at infinity of $(X, g)$ is unique.

Among the assumptions in Theorem 1.1, the Ricci-flat condition is essential since there are several examples of complete Riemannian manifolds with nonnegative Ricci curvature and Euclidean volume growth, of whom one of the tangent cones at infinity has smooth cross section, but the tangent cones at infinity is not unique [12] [7].

Here, let $\mathcal{T}(X, g)$ be the set of all of the isometry classes of the tangent cones at infinity of $(X, g)$. In this paper, the isometry between pointed metric spaces means the bijective map preserving the metrics and the base points. It is known that $\mathcal{T}(X, g)$ is closed with respect to the pointed GromovHausdorff topology, and has the natural continuous $\mathbb{R}^{+}$-action defined by the rescaling of metrics. The uniqueness of the tangent cones at infinity means that $\mathcal{T}(X, g)$ consists of only one point.

In this paper, we show that the assumption for the volume growth in Theorem 1.1 is essential. More precisely, we obtain the next main result.

Theorem 1.2. There is a complete Ricci-flat manifold $(X, g)$ of dimension 4 such that $\mathcal{T}(X, g)$ is homeomorphic to $S^{1}$. Moreover, $\mathbb{R}^{+}$-action on $\mathcal{T}(X, g)$ fixes $\left(\mathbb{R}^{3}, d_{0}^{\infty}, 0\right),\left(\mathbb{R}^{3}, h_{0}, 0\right),\left(\mathbb{R}^{3}, h_{1}, 0\right)$, where $h_{0}=\sum_{i=1}^{3}\left(d \zeta_{i}\right)^{2}$ is the Euclidean metric, $h_{1}=\frac{1}{|\zeta|} h_{0}$, and $d_{0}^{\infty}$ is the completion of the Riemannian metric

$$
\int_{0}^{\infty} \frac{d x}{\left|\zeta-\left(x^{\alpha}, 0,0\right)\right|} \cdot h_{0}
$$

and $\mathbb{R}^{+}$acts freely on

$$
\mathcal{T}(X, g) \backslash\left\{\left(\mathbb{R}^{3}, d_{0}^{\infty}, 0\right),\left(\mathbb{R}^{3}, h_{0}, 0\right),\left(\mathbb{R}^{3}, h_{1}, 0\right)\right\} .
$$

Here, $\zeta=\left(\zeta_{1}, \zeta_{2}, \zeta_{3}\right)$ is the Cartesian coordinate on $\mathbb{R}^{3}$.

Here, we mention more about the metric spaces appearing in Theorem 1.2. For $0 \leq S<T \leq \infty$, denote by $d_{S}^{T}$ the metric on $\mathbb{R}^{3}$ induced by the Riemannian metric

$$
\int_{S}^{T} \frac{d x}{\left|\zeta-\left(x^{\alpha}, 0,0\right)\right|} \cdot h_{0}
$$

For $(X, g)$ in Theorem 1.2 , we show that $\mathcal{T}(X, g)$ contains $\left\{\left(\mathbb{R}^{3}, d_{0}^{T}, 0\right) ; T \in\right.$ $\left.\mathbb{R}^{+}\right\},\left\{\left(\mathbb{R}^{3}, d_{S}^{\infty}, 0\right) ; S \in \mathbb{R}^{+}\right\}$and $\left\{\left(\mathbb{R}^{3}, h_{0}+\theta h_{1}, 0\right) ; \theta \in \mathbb{R}^{+}\right\}$. Here, we can 
check easily that $d_{0}^{T}$ and $d_{S}^{\infty}$ are homothetic to $d_{0}^{1}$ and $d_{1}^{\infty}$, respectively. We can show that

$$
\begin{aligned}
\left(\mathbb{R}^{3}, d_{0}^{T}, 0\right) \underset{T \rightarrow \infty}{\stackrel{G H}{\longrightarrow}}\left(\mathbb{R}^{3}, d_{0}^{\infty}, 0\right), & \left(\mathbb{R}^{3}, d_{0}^{T}, 0\right) \underset{T \rightarrow 0}{\stackrel{G H}{\longrightarrow}}\left(\mathbb{R}^{3}, h_{1}, 0\right), \\
\left(\mathbb{R}^{3}, d_{S}^{\infty}, 0\right) \underset{S \rightarrow \infty}{\stackrel{G H}{\longrightarrow}}\left(\mathbb{R}^{3}, h_{0}, 0\right), & \left(\mathbb{R}^{3}, d_{S}^{\infty}, 0\right) \underset{S \rightarrow 0}{\stackrel{G H}{\longrightarrow}}\left(\mathbb{R}^{3}, d_{0}^{\infty}, 0\right), \\
\left(\mathbb{R}^{3}, h_{0}+\theta h_{1}, 0\right) \underset{\theta \rightarrow \infty}{\stackrel{G H}{\longrightarrow}}\left(\mathbb{R}^{3}, h_{1}, 0\right), & \left(\mathbb{R}^{3}, h_{0}+\theta h_{1}, 0\right) \underset{\theta \rightarrow 0}{\stackrel{G H}{\longrightarrow}}\left(\mathbb{R}^{3}, h_{0}, 0\right) .
\end{aligned}
$$

Both of $\left(\mathbb{R}^{3}, h_{0}\right)$ and $\left(\mathbb{R}^{3}, h_{1}\right)$ can be regarded as the Riemannian cones with respect to the dilation $\zeta \mapsto \lambda \zeta$ on $\mathbb{R}^{3}$. Although the dilation also pulls back $d_{0}^{\infty}$ to $\lambda^{\frac{\alpha+1}{2 \alpha}} d_{0}^{\infty},\left(\mathbb{R}^{3}, d_{0}^{\infty}\right)$ does not become the metric cone with respect to this dilation since $\mathbf{l}=\left\{(t, 0,0) \in \mathbb{R}^{3} ; t \geq 0\right\}$ is not a ray. In fact, any open intervals contained in $\mathbf{l}$ have infinite length with respect to $d_{0}^{\infty}$.

In general, tangent cones at infinity of complete Riemannian manifolds with nonnegative Ricci curvature and Euclidean volume growth are metric cones [4]. In our case, it is shown in Section 9 that $\left(\mathbb{R}^{3}, d_{0}^{\infty}, 0\right)$ never become the metric cone of any metric space.

The Ricci-flat manifold $(X, g)$ appeared in Theorem 1.2 is one of the hyper-Kähler manifolds of type $A_{\infty}$, constructed by Anderson, Kronheimer and LeBrun in [1] applying Gibbons-Hawking ansatz, and by Goto in [8] as hyper-Kähler quotients. Combining Theorems 1.1 and 1.2 , we can see that the volume growth of $(X, g)$ should not be Euclidean. In fact, the author has computed the volume growth of the hyper-Kähler manifolds of type $A_{\infty}$ in [11, and showed that they are always greater than cubic growth and less than Euclidean growth. To construct $(X, g)$, we "mix" the hyper-Kähler manifold of type $A_{\infty}$ whose volume growth is $r^{a}$ for some $3<a<4$, and $\mathbb{R}^{4}$ equipped with the standard hyper-Kähler structure. Unfortunately, the author could not compute the volume growth of $(X, g)$ in Theorem 1.2 explicitly.

In this paper, we can show that a lot of metric spaces may arise as the Gromov-Hausdorff limit of hyper-Kähler manifolds of type $A_{\infty}$. Let

$$
I \in \mathcal{B}_{+}\left(\mathbb{R}^{+}\right):=\left\{J \subset \mathbb{R}^{+} ; J \text { is a Borel set of nonzero Lebesgue measure }\right\}
$$

and denote by $d_{I}$ the metric on $\mathbb{R}^{3}$ induced by the Riemannian metric $\int_{I} \frac{d x}{\left|\zeta-\left(x^{\alpha}, 0,0\right)\right|} \cdot h_{0}$. Then we have the following result.

Theorem 1.3. There is a complete Ricci-flat manifold $(X, g)$ of dimension 4 such that $\mathcal{T}(X, g)$ contains

$$
\left\{\left(\mathbb{R}^{3}, d_{I}, 0\right) ; I \in \mathcal{B}_{+}\left(\mathbb{R}^{+}\right)\right\} / \text {isometry } .
$$


Since $d_{S}^{\infty}$ and $d_{0}^{T}$ are contained in $\mathcal{T}(X, g)$ in the above theorem, then their limits $h_{0}$ and $\frac{1}{|\zeta|} h_{0}$ are also contained in $\mathcal{T}(X, g)$. The author does not know whether any other metric spaces are contained in $\mathcal{T}(X, g)$.

Theorems 1.2 and 1.3 are shown along the following process. The abovementioned hyper-Kähler manifolds are constructed from infinitely countable subsets $\Lambda$ in $\mathbb{R}^{3}$ such that $\sum_{\lambda \in \Lambda} \frac{1}{1+|\lambda|}<\infty$. We denote it by $\left(X, g_{\Lambda}\right)$ and fix the base point $p \in X$. From the construction, $\left(X, g_{\Lambda}\right)$ has a natural $S^{1}$ action preserving $g_{\Lambda}$ and the hyper-Kähler structure, then we obtain a hyperKähler moment map $\mu_{\Lambda}: X \rightarrow \mathbb{R}^{3}$ such that $\mu_{\Lambda}(p)=0$, which is a surjective map whose generic fibers are $S^{1}$. There is a unique distance function $d_{\Lambda}$ on $\mathbb{R}^{3}$ such that $\mu_{\Lambda}$ is a submetry. Here, submetries are the generalization of Riemannian submersions to the category of metric spaces. For $a>0$ we can see $a g_{\Lambda}=g_{a \Lambda}$, hence by taking $a_{i}>0$ such that $\lim _{i \rightarrow \infty} a_{i}=0$, we obtain a sequence of submetries $\mu_{a_{i} \Lambda}: X \rightarrow \mathbb{R}^{3}$. Now, assume that $\left\{\left(\mathbb{R}^{3}, d_{a_{i} \Lambda}, 0\right)\right\}_{i}$ converges to a metric space $\left(\mathbb{R}^{3}, d_{\infty}, 0\right)$ for some $d_{\infty}$ in the pointed GromovHausdorff topology, and the diameters of fibers of $\mu_{a_{i} \Lambda}$ converges to 0 in some sense. Then we can show $\left(\mathbb{R}^{3}, d_{\infty}, 0\right)$ is the Gromov-Hausdorff limit of $\left\{\left(X, g_{a_{i} \Lambda}, p\right)\right\}_{i}$. We raise a concrete example of $\Lambda$ and sequences $\left\{a_{i}\right\}_{i}$, then obtain several limit spaces. Among them, it is shown in Section 9 that $\left(\mathbb{R}^{3}, d_{0}^{\infty}\right)$ is not a polar space in the sense of Cheeger and Colding [5].

This paper is organized as follows. We review the construction of hyperKähler manifolds of type $A_{\infty}$ and hyper-Kähler moment map $\mu_{\Lambda}$ in Section 2. Then we review the notion of submetry in Section 3, and the notion of Gromov-Hausdorff topology in Section 4. In Section 5, we construct a submetry $\mu_{a}$ from $\left(X, g_{a \Lambda}\right)$ to $\left(\mathbb{R}^{3}, d_{a}\right)$ by using $\mu_{\Lambda}$ and dilation, where $d_{a}$ is the metric induced by the Riemannian metric $\Phi_{a}(\zeta) h_{0}$. Here, $\Phi_{a}$ is a positive valued harmonic function determined by $\Lambda$ and some constants. Then we see that the convergence of $\left\{\left(X, g_{a_{i} \Lambda}\right)\right\}_{i}$ can be reduced to the convergence of $\left\{\left(\mathbb{R}^{3}, d_{a_{i}}\right)\right\}_{i}$. In Sections [6] and 7 , we raise concrete examples of $\Lambda$ and fix $a>0$, then estimate the difference of $\Phi_{a}$ and another positive valued harmonic function $\Phi_{\infty}$, which induces the metric $d_{\infty}$ on $\mathbb{R}^{3}$. In Section 8 , we observe some examples by applying the results in Sections 6 and 7 , then show Theorems 1.2 and 1.3. In Section 9, we prove that $\left(\mathbb{R}^{3}, d_{0}^{\infty}\right)$ is not a polar space.

Acknowledgment. The author would like to thank Professor Shouhei Honda who invited the author to this attractive topic, and also thank him for giving the several advice on this paper. The author also would like to thank the referee for careful reading and several useful comments. Thanks to his pointing out, the author could make the main results much stronger. 
The author was supported by Grant-in-Aid for Young Scientists (B) Grant Number 16K17598.

\section{Hyper-Kähler manifolds of type $A_{\infty}$}

Here we review shortly the construction of hyper-Kähler manifolds of type $A_{\infty}$, along [1].

Let $\Lambda \subset \mathbb{R}^{3}$ be a countably infinite subset satisfying the convergence condition

$$
\sum_{\lambda \in \Lambda} \frac{1}{1+|\lambda|}<\infty
$$

and take a positive valued harmonic function $\Phi_{\Lambda}$ over $\mathbb{R}^{3} \backslash \Lambda$ defined by

$$
\Phi_{\Lambda}(\zeta):=\sum_{\lambda \in \Lambda} \frac{1}{|\zeta-\lambda|} .
$$

Then $* d \Phi_{\Lambda} \in \Omega^{2}\left(\mathbb{R}^{3} \backslash \Lambda\right)$ is a closed 2-form where $*$ is the Hodge's star operator of the Euclidean metric, and we have an integrable cohomology class $\left[\frac{1}{4 \pi} * d \Phi_{\Lambda}\right] \in H^{2}\left(\mathbb{R}^{3} \backslash \Lambda, \mathbb{Z}\right)$, which is equal to the 1st Chern class of a principal $S^{1}$-bundle $\mu=\mu_{\Lambda}: X^{*} \rightarrow \mathbb{R}^{3} \backslash \Lambda$. For every $\lambda \in \Lambda$, we can take a sufficiently small open ball $B \subset \mathbb{R}^{3}$ centered at $\lambda$ which does not contain any other elements in $\Lambda$. Then $\mu: \mu^{-1}(B \backslash\{\lambda\}) \rightarrow B \backslash\{\lambda\}$ is isomorphic to Hopf fibration $\mu_{0}: \mathbb{R}^{4} \backslash\{0\} \rightarrow \mathbb{R}^{3} \backslash\{0\}$ as principal $S^{1}$-bundles, hence there exists a $C^{\infty}$ 4-manifold $X$ and an open embedding $X^{*} \subset X$, and $\mu$ can be extended to an $S^{1}$-fibration

$$
\mu=\left(\mu_{1}, \mu_{2}, \mu_{3}\right): X \rightarrow \mathbb{R}^{3} .
$$

Moreover we may write $X \backslash X^{*}=\left\{p_{\lambda} ; \lambda \in \Lambda\right\}$ and $\mu\left(p_{\lambda}\right)=\lambda$. Next we take an $S^{1}$-connection $\Gamma \in \Omega^{1}\left(X^{*}\right)$ on $X^{*} \rightarrow \mathbb{R}^{3} \backslash \Lambda$, whose curvature form is given by $* d \Phi_{\Lambda}$. Then $\Gamma$ is uniquely determined up to exact 1 -form on $\mathbb{R}^{3} \backslash \Lambda$. Now, we obtain a Riemannian metric

$$
g_{\Lambda}:=\left(\mu^{*} \Phi_{\Lambda}\right)^{-1} \Gamma^{2}+\mu^{*} \Phi_{\Lambda} \sum_{i=1}^{3}\left(d \mu_{i}\right)^{2}
$$

on $X^{*}$, which can be extended to a smooth Riemannian metric $g_{\Lambda}$ over $X$ by taking $\Gamma$ appropriately.

Theorem $2.1([1])$. Let $\left(X, g_{\Lambda}\right)$ be as above. Then it is a complete hyperKähler (hence Ricci-flat) metric of dimension 4. 
Since $S^{1}$ acts on $\left(X, g_{\Lambda}\right)$ isometrically, it is easy to check that

$$
\mu:\left(X^{*}, g_{\Lambda}\right) \rightarrow\left(\mathbb{R}^{3} \backslash \Lambda, \Phi_{\Lambda} \cdot h_{0}\right)
$$

is a Riemannian submersion, where $h_{0}$ is the Euclidean metric on $\mathbb{R}^{3}$.

Next we consider the rescaling of $\left(X, g_{\Lambda}\right)$. For $a>0$, put $a \Lambda:=\{a \lambda \in$ $\left.\mathbb{R}^{3} ; \lambda \in \Lambda\right\}$. Then it is easy to see

$$
\Phi_{a \Lambda}(\zeta)=\sum_{\lambda \in \Lambda} \frac{1}{|\zeta-a \lambda|}=a^{-1} \sum_{\lambda \in \Lambda} \frac{1}{\left|a^{-1} \zeta-\lambda\right|}=a^{-1} \Phi_{\Lambda}\left(a^{-1} \zeta\right)
$$

and $\mu_{a \Lambda}=a \mu_{\Lambda}$, hence $\mu_{a \Lambda}^{*} \Phi_{a \Lambda}=a^{-1} \mu_{\Lambda}^{*} \Phi_{\Lambda}$ holds. Thus we have

$$
\begin{aligned}
g_{a \Lambda} & =\left(\mu_{a \Lambda}^{*} \Phi_{a \Lambda}\right)^{-1} \Gamma^{2}+\mu_{a \Lambda}^{*} \Phi_{a \Lambda} \sum_{i=1}^{3}\left(d \mu_{a \Lambda, i}\right)^{2} \\
& =a\left(\mu_{\Lambda}^{*} \Phi_{\Lambda}\right)^{-1} \Gamma^{2}+a \mu_{\Lambda}^{*} \Phi_{\Lambda} \sum_{i=1}^{3}\left(d \mu_{\Lambda, i}\right)^{2}=a g_{\Lambda} .
\end{aligned}
$$

\section{Submetry}

Throughout of this paper, the distance between $x$ and $y$ in a metric space $(X, d)$ is denoted by $d(x, y)$. If it is clear which metric is used, we often write $|x y|=d(x, y)$

The map $\mu: X \rightarrow \mathbb{R}^{3}$ appeared in the previous section is not a Riemannian submersion, since $d \mu$ degenerates on $X \backslash X^{*}$ and $\Phi_{\Lambda} \cdot h_{0}$ does not defined on the whole of $\mathbb{R}^{3}$. However we can regard $\mu$ as a submetry, which is a notion introduced in [2].

Definition $3.1([2])$. Let $X, Y$ be metric spaces, and $\mu: X \rightarrow Y$ be a map, which is not necessarily to be continuous. Then $\mu$ is said to be a submetry if $\mu(D(p, r))=D(\mu(p), r)$ holds for every $p \in X$ and $r>0$, where $D(p, r)$ is the closed ball of radius $r$ centered at $p$.

Any proper Riemannian submersions between smooth Riemannian manifolds are known to be submetries. Conversely, a submetry between smooth complete Riemannian manifolds becomes a $C^{1,1}$ Riemannian submersion [3].

Now we go back to the setting in Section 2. Denote by $d_{\Lambda}$ the metric on $\mathbb{R}^{3}$ defined as the completion of the Riemannian distance induced from $\Phi_{\Lambda} \cdot h_{0}$. Since $\mu:\left(X^{*}, g_{\Lambda}\right) \rightarrow\left(\mathbb{R}^{3} \backslash \Lambda, \Phi_{\Lambda} \cdot h_{0}\right)$ is a Riemannian submersion, we have the following proposition. 
Proposition 3.2. Let $\left(X, g_{\Lambda}\right)$ be a hyper-Kähler manifolds of type $A_{\infty}$. The map $\mu:\left(X, d_{g_{\Lambda}}\right) \rightarrow\left(\mathbb{R}^{3}, d_{\Lambda}\right)$ is a submetry, where $d_{g_{\Lambda}}$ is the Riemannian distance induced from $g_{\Lambda}$. Moreover, we have

$$
d_{\Lambda}\left(q_{0}, q_{1}\right)=\inf _{p_{1} \in \mu^{-1}\left(q_{1}\right)} d_{g_{\Lambda}}\left(p_{0}, p_{1}\right)
$$

for any $p_{0} \in \mu^{-1}\left(q_{0}\right)$

\section{The Gromov-Hausdorff convergence}

In this section, we discuss with the pointed Gromov-Hausdorff convergence of a sequence of pointed metric spaces equipped with submetries. First of all, we review the definition of the pointed Gromov-Hausdorff convergence of pointed metric spaces. Denote by $B(p, r)=B_{X}(p, r)$ the open ball of radius $r$ centered at $p$ in a metric space $X$.

Definition 4.1. Let $(X, p)$ and $\left(X^{\prime}, p^{\prime}\right)$ be pointed metric spaces, and $r, \varepsilon$ be positive real numbers. $f: B(p, r) \rightarrow X^{\prime}$ is said to be an $(r, \varepsilon)$-isometry from $(X, p)$ to $\left(X^{\prime}, p^{\prime}\right)$ if (1) $f(p)=p^{\prime}$, (2) $\|x y|-| f(x) f(y)\|<\varepsilon$ holds for any $x, y \in B(p, r),(3) B(f(B(p, r)), \varepsilon)$ contains $B\left(p^{\prime}, r-\varepsilon\right)$.

Definition 4.2. Let $\left\{\left(X_{i}, p_{i}\right)\right\}_{i}$ be a sequence of pointed metric spaces. Then $\left\{\left(X_{i}, p_{i}\right)\right\}_{i}$ is said to converge to a metric space $(X, p)$ in the pointed GromovHausdorff topology, or $\left\{\left(X_{i}, p_{i}\right)\right\}_{i} \stackrel{\mathrm{GH}}{\longrightarrow}(X, p)$, if for any $r, \varepsilon>0$ there exists an positive integer $N_{(r, \varepsilon)}$ such that $(r, \varepsilon)$-isometry from $\left(X_{i}, p_{i}\right)$ to $(X, p)$ exists for every $l \geq N_{(r, \varepsilon)}$.

For metric spaces $X, Y, q \in Y$ and a map $\mu: X \rightarrow Y$, define $\delta_{q, \mu}(r) \in$ $\mathbb{R}_{\geq 0} \cup\{\infty\}$ by

$$
\begin{aligned}
\delta_{q, \mu}(r) & :=\sup _{y \in B(q, r)} \operatorname{diam}\left(\mu^{-1}(y)\right) \\
& =\sup _{\substack{y \in B(q, r) \\
x, x^{\prime} \in \mu^{-1}(y)}}\left|x x^{\prime}\right| .
\end{aligned}
$$

Proposition 4.3. Let $\{(X, p)\}$ and $\{(Y, q)\}$ be pointed metric spaces equipped with submetries $\mu: X \rightarrow Y$ satisfying $\mu(p)=q$, and $\left(Y_{\infty}, q_{\infty}\right)$ be another pointed metric space. Assume that $\delta_{q, \mu}(r)<\infty$ and we have an $(r, \delta)$ isometry from $(Y, q)$ to $\left(Y_{\infty}, q_{\infty}\right)$. Then there exists an $\left(r, \delta+\delta_{q, \mu}\right)$-isometry from $\{(X, p)\}$ to $\left(Y_{\infty}, q_{\infty}\right)$.

Proof. Now, there is an $(r, \delta)$-isometry $f$ from $(Y, q)$ to $\left(Y_{\infty}, q_{\infty}\right)$. Then it is easy to check that the composition $\hat{f}:=f \circ \mu$ is an $\left(r, \delta+\delta_{q, \mu}\right)$-isometry from $(X, p)$ to $\left(Y_{\infty}, q_{\infty}\right)$. 


\section{$5 \quad$ Tangent cones at infinity}

Let $(X, d)$ be a metric space and $\left\{a_{i}\right\}_{i}$ be a decreasing sequence of positive numbers converging to 0 . If $(Y, q)$ is the pointed Gromov-Hausdorff limit of $\left\{\left(X, a_{i} d, p\right)\right\}_{i}$, then it is called an tangent cone at infinity of $X$. It is clear that the limit does not depend on $p \in X$, but may depend on the choice of a sequence $\left\{a_{i}\right\}_{i}$.

In this paper we are considering the tangent cones at infinity of $\left(X, d_{g_{\Lambda}}\right)$. In Section 2 we have seen $\sqrt{a} d_{g_{\Lambda}}=d_{g_{a \Lambda}}$ for $a>0$, hence $\mu_{a \Lambda}:\left(X, \sqrt{a} d_{g_{\Lambda}}\right) \rightarrow$ $\left(\mathbb{R}^{3}, d_{a \Lambda}\right)$ is a submetry. By taking $N \in \mathbb{R}^{+}$and the dilation $I_{N}: \mathbb{R}^{3} \rightarrow \mathbb{R}^{3}$ defined by $I_{N}(\zeta):=\frac{1}{N} \zeta$, we have another submetry

$$
\mu_{a}:=I_{N}^{-1} \circ \mu_{a \Lambda}:\left(X, \sqrt{a} d_{g_{\Lambda}}\right) \rightarrow\left(\mathbb{R}^{3}, d_{a}:=I_{N}{ }^{*} d_{a \Lambda}\right) .
$$

Here, $I_{N}{ }^{*} d_{a \Lambda}$ is the completion of the Riemannian distance of

$$
I_{N}{ }^{*}\left(\Phi_{a \Lambda} \cdot h_{0}\right)=I_{N}{ }^{*} \Phi_{a \Lambda} \cdot \frac{1}{N^{2}} h_{0}=N \Phi_{N a \Lambda} \cdot \frac{1}{N^{2}} h_{0}=\frac{1}{N} \Phi_{N a \Lambda} \cdot h_{0},
$$

therefore we obtain $d_{a}$ which is the completion of the Riemannian metric $\Phi_{a} \cdot h_{0}$, where

$$
\Phi_{a}:=\frac{1}{N} \Phi_{N a \Lambda}
$$

In other words, $d_{a}$ is given by

$$
d_{a}(x, y)=\inf _{\gamma \in \operatorname{Path}(x, y)} l_{a}(\gamma)
$$

where $\operatorname{Path}(x, y)$ is the set of smooth paths in $\mathbb{R}^{3}$ joining $x, y \in \mathbb{R}^{3}$, and

$$
l_{a}(\gamma)=\int_{t_{0}}^{t_{1}} \sqrt{\Phi_{a}(\gamma(t))}\left|\gamma^{\prime}(t)\right| d t
$$

By the definition of $g_{\Lambda}$, one can see that the diameter of the fiber $\mu_{\Lambda}^{-1}(\zeta)$ is given by $\frac{\pi}{\sqrt{\Phi_{\Lambda}(\zeta)}}$. Accordingly, the diameter of $\mu_{a}^{-1}(\zeta)$ is given by $\frac{\pi}{N \sqrt{\Phi_{a}(\zeta)}}$.

For a metric $d_{\infty}$ on $\mathbb{R}^{3}$ and constants $r, \delta, \delta^{\prime}>0$, we introduce the next assumptions.

(A1) The identity map

$$
\operatorname{id}_{\mathbb{R}^{3}}:\left(\mathbb{R}^{3}, d_{a}, 0\right) \rightarrow\left(\mathbb{R}^{3}, d_{\infty}, 0\right)
$$

is an $(r, \delta)$-isometry. 
(A2) $\sup _{\zeta \in B_{d_{a}}(0, r)} \frac{\pi}{N \sqrt{\Phi_{a}(\zeta)}}<\delta^{\prime}$ holds.

Then we obtain the next proposition by Proposition 4.3 ,

Proposition 5.1. Let $\left(X, g_{\Lambda}\right)$ and $\mu_{a}$ be as above, $p \in X$ satisfy $\mu_{\Lambda}(p)=$ 0 and $d_{\infty}$ be a metric on $\mathbb{R}^{3}$. If (A1-2) are satisfied for given constants $r, \delta, \delta^{\prime}>0$, then $\mu_{a}$ is an $\left(r, \delta+\delta^{\prime}\right)$-isometry from $\left(X, a g_{\Lambda}, p\right)$ to $\left(\mathbb{R}^{3}, d_{\infty}, 0\right)$.

\section{Construction}

Fix $\alpha>1$, and let

$$
\Lambda^{\alpha}:=\left\{\left(k^{\alpha}, 0,0\right) ; k \in \mathbb{Z}_{\geq 0}\right\} .
$$

Take an increasing sequence of integers $0<K_{0}<K_{1}<K_{2}<\cdots$.

In this paper many constants will appear, and they may depend on $\alpha$ or $\left\{K_{n}\right\}$. However, we do not mind the dependence on these parameters.

Put

$$
\begin{aligned}
\Lambda_{2 n} & :=\left\{\left(k^{\alpha}, 0,0\right) \in \Lambda^{\alpha} ; K_{2 n} \leq k<K_{2 n+1}\right\}, \\
\Lambda & :=\bigcup_{n=0}^{\infty} \Lambda_{2 n} .
\end{aligned}
$$

Since $\Lambda \subset \Lambda^{\alpha}$, we can see that $\sum_{\lambda \in \Lambda} \frac{1}{1+|\lambda|}<\infty$, accordingly we obtain a hyper-Kähler manifold $\left(X, g_{\Lambda}\right)$.

From now on we fix $a>0, n \in \mathbb{N}$ and $P>0$, then put $N:=a^{\frac{-1}{1+\alpha}} P^{\frac{1}{1+\alpha}}$ and

$$
\Phi_{a}(\zeta):=\frac{1}{N} \Phi_{N a \Lambda}(\zeta)=\sum_{\lambda \in \Lambda} \frac{1}{N\left|\zeta-P N^{-\alpha} \lambda\right|}
$$

Let $\mathbf{l}:=\left\{(t, 0,0) \in \mathbb{R}^{3} ; t \geq 0\right\}$ and put

$$
K(R, D):=\left\{\zeta \in \mathbb{R}^{3} ;|\zeta| \leq R, \inf _{y \in \mathbf{l}}|\zeta-y| \geq D\right\}
$$

Here, $\inf _{y \in \mathbf{l}}|\zeta-y|$ is given by

$$
\inf _{y \in \mathbf{l}}|\zeta-y|= \begin{cases}\sqrt{\mid \zeta_{\left.\mathbb{C}\right|^{2}}} & \left(\text { if } \zeta_{\mathbb{R}} \geq 0\right) \\ |\zeta| & \text { (if } \left.\zeta_{1}<0\right)\end{cases}
$$


for $\zeta=\left(\zeta_{\mathbb{R}}, \zeta_{\mathbb{C}}\right) \in \mathbb{R}^{3}=\mathbb{R} \oplus \mathbb{C}$. For $0 \leq S<T \leq \infty$, define a positive valued function $\Phi_{S, P}^{T}: \mathbb{R}^{3} \backslash \mathbf{l}: \rightarrow \mathbb{R}$ by

$$
\Phi_{S, P}^{T}(\zeta):=\int_{S}^{T} \frac{d x}{\left|\zeta-P\left(x^{\alpha}, 0,0\right)\right|} .
$$

Throughout this section, we put

$$
S_{n}:=\frac{K_{2 n}}{N}=a^{\frac{1}{1+\alpha}} P^{\frac{-1}{1+\alpha}} K_{2 n}, \quad T_{n}:=\frac{K_{2 n+1}}{N}=a^{\frac{1}{1+\alpha}} P^{\frac{-1}{1+\alpha}} K_{2 n+1} .
$$

Proposition 6.1. We have

$$
\left|\Phi_{a}(\zeta)-\sum_{n=0}^{\infty} \Phi_{S_{n}, P}^{T_{n}}(\zeta)\right| \leq \frac{2}{N D}=\frac{2}{D}\left(\frac{a}{P}\right)^{\frac{1}{1+\alpha}}
$$

for any $\zeta \in K(R, D)$.

Proof. Since

$$
\Lambda_{2 n}=\left\{\left(k^{\alpha}, 0,0\right) ; K_{2 n} \leq k<K_{2 n+1}\right\}
$$

we have

$$
\sum_{\lambda \in \Lambda_{2 n}} \frac{1}{N\left|\zeta-P N^{-\alpha} \lambda\right|}=\sum_{k=K_{2 n}}^{K_{2 n+1}-1} \frac{1}{N\left|\zeta-P N^{-\alpha}\left(k^{\alpha}, 0,0\right)\right|}
$$

Then we obtain

$$
\left|\sum_{n=0}^{\infty}\left(\sum_{\lambda \in \Lambda_{2 n}} \frac{1}{N\left|\zeta-P N^{-\alpha} \lambda\right|}-\int_{K_{2 n} / N}^{K_{2 n+1} / N} \frac{d x}{\left|\zeta-P\left(x^{\alpha}, 0,0\right)\right|}\right)\right| \leq \frac{2}{N D} .
$$

The above inequality holds since the function $x \mapsto \frac{1}{\left|\zeta-P\left(x^{\alpha}, 0,0\right)\right|}$ has at most one critical point and

$$
\sup _{x \in \mathbb{R}} \frac{1}{\left|\zeta-P\left(x^{\alpha}, 0,0\right)\right|}-\inf _{x \in \mathbb{R}} \frac{1}{\left|\zeta-P\left(x^{\alpha}, 0,0\right)\right|} \leq \frac{1}{D}
$$

for all $\zeta \in K(R, D)$.

Next we obtain the lower estimate of $\Phi_{a}$ as follows. 
Proposition 6.2. We have

$$
\begin{aligned}
\Phi_{S_{n}, P}^{T_{n}}(\zeta) & \geq\left(\int_{S_{n}}^{T_{n}} \frac{d x}{1+P x^{\alpha}}\right) \min \left\{\frac{1}{|\zeta|}, 1\right\}, \\
\Phi_{a}(\zeta) & \geq\left(\sum_{n=0}^{\infty} \int_{S_{n}}^{T_{n}} \frac{d x}{1+P x^{\alpha}}-2\left(a P^{-1}\right)^{\frac{1}{1+\alpha}}\right) \min \left\{\frac{1}{|\zeta|}, 1\right\}, \\
\sum_{n=0}^{\infty} \Phi_{S_{n}, P}^{T_{n}}(\zeta) & \leq P^{-\frac{1}{\alpha}} \frac{\alpha 2^{\frac{1}{\alpha}}}{\alpha-1} \frac{|\zeta|^{\frac{1}{\alpha}}}{\mid \zeta_{\mathbb{C} \mid}} \\
\sum_{n=n_{0}}^{\infty} \Phi_{S_{n}, P}^{T_{n}}(\zeta) & \leq \frac{2 S_{n_{0}}^{-\alpha+1}}{P(\alpha-1)} \quad\left(\text { if } S_{n_{0}} \geq\left(\frac{2|\zeta|}{P}\right)^{\frac{1}{\alpha}}\right), \\
\sum_{n=0}^{n_{0}} \Phi_{S_{n}, P}^{T_{n}}(\zeta) & \left.\leq \frac{T_{n_{0}}}{D} \quad \text { (if } \zeta \in K(R, D)\right) .
\end{aligned}
$$

Proof. First of all one can see

$$
\Phi_{S_{n}, P}^{T_{n}}(\zeta) \geq \int_{S_{n}}^{T_{n}} \frac{d x}{|\zeta|+P x^{\alpha}} \geq \frac{1}{|\zeta|} \int_{S_{n}}^{T_{n}} \frac{d x}{1+P x^{\alpha}}
$$

if $|\zeta| \geq 1$, and

$$
\Phi_{S_{n}, P}^{T_{n}}(\zeta) \geq \int_{S_{n}}^{T_{n}} \frac{d x}{|\zeta|+P x^{\alpha}} \geq \int_{S_{n}}^{T_{n}} \frac{d x}{1+P x^{\alpha}}
$$

if $|\zeta| \leq 1$.

Next we have

$$
\Phi_{a}(\zeta) \geq \sum_{n=0}^{\infty} \sum_{k=K_{2 n}}^{K_{2 n+1}-1} \frac{1}{N\left(|\zeta|+P N^{-\alpha} k^{\alpha}\right)}
$$

and the similar argument to the proof of Proposition 6.1 gives

$$
\left|\sum_{n=0}^{\infty}\left(\sum_{k=K_{2 n}}^{K_{2 n+1}-1} \frac{1}{N\left(|\zeta|+P N^{-\alpha} k^{\alpha}\right)}-\int_{S_{n}}^{T_{n}} \frac{d x}{|\zeta|+P x^{\alpha}}\right)\right| \leq \frac{2}{N|\zeta|} .
$$

Combining these inequalities one can the second assertion if $|\zeta| \geq 1$. If $|\zeta| \leq 1$, then we have

$$
\Phi_{a}(\zeta) \geq \sum_{n=0}^{\infty} \sum_{k=K_{2 n}}^{K_{2 n+1}-1} \frac{1}{N\left(1+P N^{-\alpha} k^{\alpha}\right)}
$$


and by the similar argument we obtain the assertion.

Next we consider ([6). If $t \geq\left(\frac{2|\zeta|}{P}\right)^{\frac{1}{\alpha}}$, then

$$
\int_{t}^{\infty} \frac{d x}{\left|\zeta-P\left(x^{\alpha}, 0,0\right)\right|} \leq \int_{t}^{\infty} \frac{2 d x}{P x^{\alpha}}=\frac{2}{P(\alpha-1)} t^{-\alpha+1}
$$

holds. Hence one can see

$$
\begin{aligned}
\sum_{n=0}^{\infty} \Phi_{S_{n}, P}^{T_{n}}\left(\zeta_{\mathbb{R}}, \zeta_{\mathbb{C}}\right) & \leq \int_{0}^{\infty} \frac{d x}{\left|\zeta-P\left(x^{\alpha}, 0,0\right)\right|} \\
& =\int_{0}^{\left(\frac{2|\zeta|}{P}\right)^{\frac{1}{\alpha}}} \frac{d x}{\left|\zeta-P\left(x^{\alpha}, 0,0\right)\right|}+\int_{\left(\frac{2|\zeta|}{P}\right)^{\frac{1}{\alpha}}}^{\infty} \frac{d x}{\left|\zeta-P\left(x^{\alpha}, 0,0\right)\right|} \\
& \leq \frac{\left(\frac{2|\zeta|}{P}\right)^{\frac{1}{\alpha}}}{\mid \zeta_{\mathbb{C}}}+\frac{2}{P(\alpha-1)}\left(\frac{2|\zeta|}{P}\right)^{\frac{1}{\alpha}(-\alpha+1)} \\
& =P^{-\frac{1}{\alpha}}\left(\frac{(2|\zeta|)^{\frac{1}{\alpha}}}{\left|\zeta_{\mathbb{C}}\right|}+\frac{(2|\zeta|)^{\frac{1}{\alpha}}}{\alpha-1} \frac{1}{\left|\zeta_{\mathbb{C}}\right|}\right)
\end{aligned}
$$

We have (7) by (91). (8) is obvious.

Put

$$
A_{S, P}^{T}:=\int_{S}^{T} \frac{d x}{1+P x^{\alpha}}
$$

By Proposition 6.2, we have the following.

Proposition 6.3. Let $\Phi_{a}$ be as above. Then

$$
\sup _{|\zeta| \leq R} \frac{1}{N \sqrt{\Phi_{a}(\zeta)}} \leq\left(\frac{a}{P}\right)^{\frac{1}{1+\alpha}}\left(\sum_{n=0}^{\infty} A_{S_{n}, P}^{T_{n}}-2\left(\frac{a}{P}\right)^{\frac{1}{1+\alpha}}\right)^{-\frac{1}{2}} \sqrt{R}
$$

holds for every $R \geq 1$.

\section{Distance}

In the previous section we have estimated $\left|\Phi_{a}-\sum_{n} \Phi_{S_{n}, P}^{T_{n}}\right|$ from the above on $K(R, D)$.

In this section we introduce more general positive functions $\Phi$ and $\Phi_{\infty}$, and induced metric $d, d_{\infty}$ on $\mathbb{R}^{3}$ respectively. What we hope to show in this section is that if we fix a very large $R \geq 1$ and assume that $\sup _{K(R, D)} \mid \Phi-$ $\Phi_{\infty} \mid \leq \frac{\varepsilon}{D}$ holds for a very small $\varepsilon$ and every $D \leq 1$, then the identity map 
of $\mathbb{R}^{3}$ becomes the $(r, \delta)$-isometry from $\left(\mathbb{R}^{3}, d, 0\right)$ to $\left(\mathbb{R}^{3}, d_{\infty}, 0\right)$, for a large $r$ and a small $\delta$. Here, we explain the difficulty to show it.

We hope to show that $\sup _{K(R, D)}\left|d-d_{\infty}\right|$ is small for every $R \geq 1$ and $0<D \leq 1$. By the estimate of $\sup _{K(R, D)}\left|\Phi-\Phi_{\infty}\right|$, it is easy to see that $\sup _{K(R, D)}\left|d_{R, D}-d_{\infty, R, D}\right|$ is small, where $d_{R, D}$ (resp. $\left.d_{\infty, R, D}\right)$ is the Riemannian distance of the Riemannian metric $\left.\Phi h_{0}\right|_{K(R, D)}$ (resp. $\left.\left.\Phi_{\infty} h_{0}\right|_{K(R, D)}\right)$. However, $d_{R, D}$ may not equal to $d$ in general since the geodesic of $\Phi h_{0}$ joining two points in $K(R, D)$ might leave from $K(R, D)$. To see that $\sup _{K(R, D)}\left|d_{R, D}-d\right|$ is sufficiently small, we have to observe that a path joining two points in $K(R, D)$ which leaves $K(R, D)$ can be replaced by a shorter path included in $K(R, D)$.

In this section we consider positive valued functions $\Phi, \Phi_{\infty} \in C^{\infty}\left(\mathbb{R}^{3} \backslash \mathbf{l}\right)$ satisfying the following conditions for given constants $R \geq 1, m, \varepsilon, C_{0}, C_{1}>0$ and $\kappa \geq 0$.

(A3).

$$
\begin{aligned}
& \left|\Phi(\zeta)-\Phi_{\infty}(\zeta)\right| \leq \frac{\varepsilon}{D^{m}} \\
& \left|\Phi(\zeta)-\Phi_{\infty}(\zeta)\right| \leq \frac{C_{1}}{D}
\end{aligned}
$$

holds for any $D \leq 1$ and $\zeta \in K(R, D)$.

(A4). Along the decomposition $\mathbb{R}^{3}=\mathbb{R} \oplus \mathbb{C}$, put $\zeta=\left(\zeta_{\mathbb{R}}, \zeta_{\mathbb{C}}\right) \in \mathbb{R} \oplus \mathbb{C}$. Then

$$
\begin{aligned}
\Phi\left(\zeta_{\mathbb{R}}, e^{i \theta} \zeta_{\mathbb{C}}\right) & =\Phi\left(\zeta_{\mathbb{R}}, \zeta_{\mathbb{C}}\right), \quad \Phi\left(\zeta_{\mathbb{R}}, \zeta_{\mathbb{C}}\right) \leq \Phi\left(\zeta_{\mathbb{R}}, \zeta_{\mathbb{C}}^{\prime}\right) \\
\Phi_{\infty}\left(\zeta_{\mathbb{R}}, e^{i \theta} \zeta_{\mathbb{C}}\right) & =\Phi_{\infty}\left(\zeta_{\mathbb{R}}, \zeta_{\mathbb{C}}\right), \quad \Phi_{\infty}\left(\zeta_{\mathbb{R}}, \zeta_{\mathbb{C}}\right) \leq \Phi_{\infty}\left(\zeta_{\mathbb{R}}, \zeta_{\mathbb{C}}^{\prime}\right)
\end{aligned}
$$

holds for any $e^{i \theta} \in S^{1}$, if $\left|\zeta_{\mathbb{C}}\right| \geq\left|\zeta_{\mathbb{C}}^{\prime}\right|$.

(A5).

$$
\min \left\{\Phi(\zeta), \Phi_{\infty}(\zeta)\right\} \geq \begin{cases}\frac{C_{0}}{|\zeta|} & (\text { if }|\zeta| \geq 1) \\ C_{0} & (\text { if }|\zeta| \leq 1)\end{cases}
$$

(A6). For any $u \geq 1$ and $\zeta \in \mathbb{R}^{3} \backslash \mathbf{l}$ with $|\zeta| \leq u$,

$$
\Phi_{\infty}(\zeta) \leq \frac{C_{1} u^{\kappa}}{\left|\zeta_{\mathbb{C}}\right|}
$$

holds. 
Remark 7.1. Let $\Phi=\Phi_{a}$ and $\Phi_{\infty}=\Phi_{S, P}^{T}$ be as in Section 6, Then they satisfy (A4), and also satisfy (A3)(A5)(A6) for appropriate constants $\varepsilon, C_{0}, C_{1}$ given by Propositions 6.1 and 6.2.

From now on, let $\Phi, \Phi_{\infty}$ satisfy (A3-6) for constants $R, \varepsilon, C_{0}, C_{1}, \kappa$. Denote by $d, d_{\infty}$ the metric on $\mathbb{R}^{3}$ induced by $\Phi \cdot h, \Phi_{\infty} \cdot h$, and by $l, l_{\infty}$ the length of the path with respect to $d, d_{\infty}$, respectively.

\subsection{Estimates (1)}

Let $\mathbf{B}(u):=\left\{\zeta \in \mathbb{R}^{3} ;|\zeta|<u\right\}$ and $\operatorname{Path}(u, x, y)$ be the set of smooth paths in $\overline{\mathbf{B}(u)}$ joining $x, y \in \overline{\mathbf{B}(u)}$, then put

$$
\begin{aligned}
d_{u}(x, y) & =\inf _{\gamma \in \operatorname{Path}(u, x, y)} l(\gamma), \\
d_{\infty, u}(x, y) & =\inf _{\gamma \in \operatorname{Path}(u, x, y)} l_{\infty}(\gamma),
\end{aligned}
$$

for $r$. By the definition, $d(x, y) \leq d_{u}(x, y)$ and $d_{\infty}(x, y) \leq d_{\infty, u}(x, y)$ always hold. However, the opposite inequality may not hold, since the minimizing geodesic $\gamma$ joining $x, y \in \overline{\mathbf{B}(u)}$ may leave from $\overline{\mathbf{B}(u)}$. The goal of this subsection is to show $d_{\rho(u)}(x, y) \leq d(x, y)$ and $d_{\infty, \rho(u)}(x, y) \leq d_{\infty}(x, y)$ for a sufficiently large $\rho(u)$.

Proposition 7.2. Suppose $\Phi, \Phi_{\infty}$ satisfy (A3-6). Let $D_{u}$ and $D_{u, u^{\prime}}$ be the diameters of $\overline{\mathbf{B}(t)}$ with respect to $d$ and $d_{u^{\prime}}$ respectively, where $0<u \leq u^{\prime}$. Define $D_{\infty, u}$ and $D_{\infty, u, u^{\prime}}$ in the same way. Then the inequality

$$
2 \sqrt{C_{0}}(\sqrt{|\zeta|}-1) \leq \min \left\{d(0, \zeta), d_{\infty}(0, \zeta)\right\}
$$

holds for all $\zeta \in \mathbb{R}^{3}$, and

$$
\begin{aligned}
d(0, \zeta) & \leq D_{u} \leq D_{u, u} \leq C_{2} u^{\kappa^{\prime}} \\
d_{\infty}(0, \zeta) & \leq D_{\infty, u} \leq D_{\infty, u, u} \leq C_{2} u^{\kappa^{\prime}}
\end{aligned}
$$

hold for all $\zeta \in \mathbb{R}^{3}, u \geq 1$ with $|\zeta| \leq u \leq R$, where $C_{2}$ is the constant depending only on $C_{1}$ and $\kappa^{\prime}=\frac{1+\kappa}{2}$.

Proof. First of all we show the first inequality. Let $\gamma:[a, b] \rightarrow \mathbb{R}^{3}$ be a smooth path such that $\gamma(a)=0$ and $\gamma(b)=\zeta$. We may suppose $|\zeta| \geq 1$, since it is obviously satisfied when $|\zeta|<1$. Then there is $s \in[a, b]$ such that $|\gamma(s)|=1$ and $|\gamma(t)| \geq 1$ for any $t \in[s, b]$. Then by the assumption (A5), one can see

$$
l(\gamma)=\int_{a}^{b} \sqrt{\Phi(\gamma(t))}\left|\gamma^{\prime}(t)\right| d t \geq \int_{s}^{b} \sqrt{\Phi(\gamma)}\left|\gamma^{\prime}\right| d t \geq \int_{s}^{b} \sqrt{\frac{C_{0}}{|\gamma|}}\left|\gamma^{\prime}\right| d t
$$


Since we have $\left|\gamma^{\prime}\right| \geq|\gamma|^{\prime}$ holds, we obtain

$$
l(\gamma) \geq \int_{s}^{b} \sqrt{\frac{C_{0}}{|\gamma|}}|\gamma|^{\prime} d t \geq 2 \sqrt{C_{0}} \int_{s}^{b} \frac{d}{d t} \sqrt{|\gamma|} d t \geq 2 \sqrt{C_{0}}(\sqrt{|\zeta|}-1)
$$

for all $\zeta \in \mathbb{R}^{3}$ with $|\zeta| \geq 1$.

By the definition, $d(0, \zeta) \leq D_{u} \leq D_{u, R_{1}} \leq D_{u, R_{0}}$ always hold for any $u \leq R_{0} \leq R_{1}$ and $\zeta \in \mathbb{R}^{3}$ with $|\zeta| \leq u$. Next we estimate $D_{u, u}$ from the above. For every $\zeta$, we prepare the piecewise smooth paths $\gamma_{\zeta}$ in $\overline{\mathbf{B}(u)}$ joining 0 and $\zeta$ as follows. Then we have an upper bound

$$
D_{u, u} \leq 2 \sup _{\zeta \in \overline{\mathbf{B}(u)}} l\left(\gamma_{\zeta}\right)
$$

Here we define $\gamma_{\zeta}$ as follows. Now we have the isometric $S^{1}$-action on $\mathbb{R}^{3}$ with respect to $d, d_{\infty}$ by (A4). By supposing $\gamma_{e^{i \theta} \zeta}=e^{i \theta} \gamma_{\zeta}$, it suffices to consider $\gamma_{\zeta}$ in the case of $\zeta=r(\sin s,-\cos s, 0)$, where $r>0$ and $-\pi<s \leq \pi$. Let

$$
\begin{aligned}
\left.\gamma_{\zeta}\right|_{[0,1]}(t) & :=(0,-r t, 0), \\
\left.\gamma_{\zeta}\right|_{[1,2]}(t) & :=r(\sin \{s(t-1)\},-\cos \{s(t-1)\}, 0) .
\end{aligned}
$$

Since $\zeta \in K\left(R,\left|\zeta_{\mathbb{C}}\right|\right)$ holds, (A3) gives $\left|\Phi(\zeta)-\Phi_{\infty}(\zeta)\right| \leq \frac{C_{1}}{\mid \zeta_{\mathbb{C}}}$, and (A6) gives $\Phi_{\infty}(\zeta) \leq C_{1} u^{\kappa} /\left|\zeta_{\mathbb{C}}\right|$. Then we can see

$$
\begin{aligned}
l\left(\left.\gamma_{\zeta}\right|_{[0,1]}\right) & =\int_{0}^{1} \sqrt{\Phi\left(\gamma_{\zeta}\right)}\left|\gamma_{\zeta}^{\prime}\right| d t \\
& \leq \int_{0}^{1} r \sqrt{\left|\Phi\left(\gamma_{\zeta}\right)-\Phi_{\infty}\left(\gamma_{\zeta}\right)\right|} d t+\int_{0}^{1} r \sqrt{\left|\Phi_{\infty}\left(\gamma_{\zeta}\right)\right|} d t \\
& \leq \int_{0}^{1} r \sqrt{\frac{C_{1}}{r t}} d t+\int_{0}^{1} r \sqrt{\frac{C_{1} u^{\kappa}}{r t}} d t \\
& \leq 2 \sqrt{C_{1} u}+2 \sqrt{C_{1}} u^{\frac{\kappa+1}{2}} .
\end{aligned}
$$

Simultaneously, we also have

$$
\begin{aligned}
l\left(\left.\gamma_{\zeta}\right|_{[1,2]}\right) & \leq \int_{1}^{2}\left|\gamma_{\zeta}^{\prime}\right| \sqrt{\left|\Phi\left(\gamma_{\zeta}\right)-\Phi_{\infty}\left(\gamma_{\zeta}\right)\right|} d t+\int_{1}^{2}\left|\gamma_{\zeta}^{\prime}\right| \sqrt{\left|\Phi_{\infty}\left(\gamma_{\zeta}\right)\right|} d t \\
& \leq \int_{0}^{1} r|s| \sqrt{\frac{C_{1}}{r|\cos s t|}} d t+\int_{0}^{1} r|s| \sqrt{\frac{C_{1} u^{\kappa}}{r|\cos s t|}} d t \\
& \leq \sqrt{C_{1} u}+\sqrt{C_{1} u^{1+\kappa}} \int_{0}^{|s|} \sqrt{\frac{1}{\cos t}} d t .
\end{aligned}
$$


Here, $\int_{0}^{\pi} \sqrt{\frac{1}{\cos t}} d t$ is finite. Since $u \geq 1$ and $\kappa \geq 0$, we may suppose $\max \left\{\sqrt{u}, \sqrt{u^{1+\kappa}}\right\}=u^{1+\kappa}$. By combining these estimates and putting $C_{2}=$ $\left(2+\int_{0}^{\pi} \sqrt{\frac{1}{\cos t}} d t\right) \sqrt{C_{1}}$, we have the assertion. The estimate of $D_{\infty, u, u}$ also obtained by the above argument.

Proposition 7.3. Suppose $\Phi, \Phi_{\infty}$ satisfy (A3-6), and let

$$
\rho(t):=\max \left\{t-1,\left(1+C_{3} t^{\kappa^{\prime}}\right)^{2}\right\},
$$

for $t>0$, where $C_{3}=\frac{3 C_{2}}{2 \sqrt{C_{0}}}$ and $C_{2}$ is the constant in Proposition 7.2. Then $d_{\rho(u)}(x, y)=d(x, y)$ and $d_{\infty, \rho(u)}(x, y)=d_{\infty}(x, y)$ holds for any $x, y \in \mathbf{B}(u)$ and $1 \leq u \leq R$.

Proof. By the definition, $d(x, y) \leq d_{\rho(u)}(x, y)$ always holds. We assume $d(x, y)<d_{\rho(u)}(x, y)$ for some $x, y \in \mathbf{B}(u)$. Then there is a smooth $\gamma:$ $[a, b] \rightarrow \mathbb{R}^{3}$ joining $x$ and $y$ such that $d(x, y) \leq l(\gamma)<d_{\rho(u)}(x, y)$, which implies the existence of $c \in[a, b]$ satisfying $|\gamma(c)|=\rho(u)$. Then one can see

$$
\begin{aligned}
l(\gamma) \geq l\left(\left.\gamma\right|_{[a, c]}\right) & \geq d(0, \gamma(c))-d(0, \gamma(a)) \\
& \geq 2 \sqrt{C_{0}}(\sqrt{\rho(u)}-1)-D_{u, u} \\
& \geq 2 \sqrt{C_{0}}\left(\sqrt{\left(1+C_{3} u^{\kappa^{\prime}}\right)^{2}}-1\right)-C_{2} u^{\kappa^{\prime}} \\
& \geq 2 C_{2} u^{\kappa^{\prime}}
\end{aligned}
$$

by Proposition 7.2. On the other hand, we have

$$
d_{\rho(u)}(x, y) \leq D_{u, \rho(u)} \leq D_{u, u} \leq C_{2} u^{\kappa^{\prime}}
$$

by Proposition 7.2. Therefore we obtain

$$
2 C_{2} u^{\kappa^{\prime}} \leq l(\gamma)<d_{\rho(u)}(x, y) \leq C_{2} u^{\kappa^{\prime}},
$$

we have a contradiction. $d_{\infty}(x, y)=d_{\infty, \rho(u)}(x, y)$ is also shown in the same way.

\subsection{Estimates (2)}

In this subsection, let $\gamma:[a, b] \rightarrow \mathbf{B}(u)$ be a smooth path joining $x, y \in$ $\mathbb{R}^{3} \backslash L(D)$ where

$$
L(D):=\left\{\zeta \in \mathbb{R}^{3} ;\left|\zeta_{\mathbb{C}}\right|<D\right\} .
$$

Now, we are going to show that if $\gamma$ is a minimizing geodesic joining $x$ and $y$, then it never approaches to the axis $\left\{(t, 0,0) \in \mathbb{R}^{3} ; t \in \mathbb{R}\right\}$. To show it, if the given $\gamma$ invade $L(D)$, then we modify $\gamma$ and construct the new path $c_{\gamma}$ not to invade $L(D)$. 
Lemma 7.4. Suppose $\Phi, \Phi_{\infty}$ satisfy (A4). Let $\gamma=\left(\gamma_{\mathbb{R}}, \gamma_{\mathbb{C}}\right):[a, b] \rightarrow \mathbb{R}^{3}=$ $\mathbb{R} \oplus \mathbb{C}$ be a smooth path satisfying that $\left|\gamma_{\mathbb{C}}(a)\right|=\left|\gamma_{\mathbb{C}}(b)\right|=D$ and $\left|\gamma_{\mathbb{C}}(t)\right| \leq D$ for any $t \in[a, b]$. Define $P_{\gamma}:[a, b] \rightarrow \mathbb{R}^{3}$ by

$$
P_{\gamma}(t):=\left(\gamma_{\mathbb{R}}(t), \gamma_{\mathbb{C}}(a)\right)
$$

Then $l\left(P_{\gamma}\right) \leq l(\gamma)$ and $l_{\infty}\left(P_{\gamma}\right) \leq l_{\infty}(\gamma)$ hold

Proof. Since $\Phi(\gamma(t)) \geq \Phi\left(P_{\gamma}(t)\right)$ holds by the second inequality of (A4), and

$$
\left|\gamma^{\prime}\right|^{2}=\left|\gamma_{\mathbb{R}}^{\prime}\right|^{2}+\left|\gamma_{\mathbb{C}}^{\prime}\right|^{2} \geq\left|\gamma_{\mathbb{R}}^{\prime}\right|^{2}=\left|P_{\gamma}^{\prime}\right|^{2}
$$

holds, we can deduce

$$
l(\gamma)=\int_{a}^{b} \sqrt{\Phi(\gamma(t))}\left|\gamma^{\prime}(t)\right| d t \geq \int_{a}^{b} \sqrt{\Phi\left(P_{\gamma}(t)\right)}\left|P_{\gamma}^{\prime}(t)\right| d t . \geq l\left(P_{\gamma}\right) .
$$

Let $\gamma:[a, b] \rightarrow \mathbb{R}^{3}$ be a smooth path joining $x, y \in \mathbb{R}^{3} \backslash L(D)$, and assume that $\left|\gamma_{\mathbb{C}}\left(a^{\prime}\right)\right|=\left|\gamma_{\mathbb{C}}\left(b^{\prime}\right)\right|=D$ and $\gamma\left(\left(a^{\prime}, b^{\prime}\right)\right)$ is contained in $\overline{L(D)}$ for some $a \leq a^{\prime}<b^{\prime} \leq b$. Then define a new path $\Gamma\left(\gamma,\left[a^{\prime}, b^{\prime}\right]\right):[a, b] \rightarrow \mathbb{R}^{3}$ by connecting

$$
\left.\gamma\right|_{\left[a, a^{\prime}\right]}, \quad P_{\left.\gamma\right|_{\left[a^{\prime}, b^{\prime}\right]},},\left.\quad e^{i \theta} \gamma\right|_{\left[b^{\prime}, b\right]}
$$

Here, by choosing $e^{i \theta}$ appropriately, $\Gamma\left(\gamma,\left[a^{\prime}, b^{\prime}\right]\right)$ is the continuous and piecewise smooth. By Lemma 7.4 , the length of $\Gamma\left(\gamma,\left[a^{\prime}, b^{\prime}\right]\right)$ is not longer than that of $\gamma$ since $S^{1}$ rotation preserves $d$ and $d_{\infty}$.

Put $J:=\gamma^{-1}(L(D)) \cap(a, b)$. Since $J$ is open in $(a, b)$, it is decomposed into the disjoint open intervals such as

$$
J=\bigsqcup_{q \in \mathcal{Q}}\left(a_{q}, b_{q}\right)
$$

respectively for some $a_{q}, b_{q} \in[a, b]$ and countable sets $\mathcal{Q}$. If $q \in \mathcal{Q}$, then $\left|\gamma_{\mathbb{C}}\left(a_{q}\right)\right|=\left|\gamma_{\mathbb{C}}\left(b_{q}\right)\right|=D$ holds. Then we have $\gamma_{1}:=\Gamma\left(\gamma,\left[a_{q}, b_{q}\right]\right)$ for a fixed $q \in \mathcal{Q}$, moreover we obtain $\gamma_{2}:=\Gamma\left(\gamma_{1},\left[a_{q^{\prime}}, b_{q}\right]\right)$ for another $q^{\prime} \in \mathcal{Q}$, and repeating this process for all $q \in \mathcal{Q}$ we finally obtain the piecewise smooth path $c:[a, b] \rightarrow \mathbb{R}^{3}$ such that $c(a)=\gamma(a), c(b)=e^{i \theta} \gamma(b)$ for some $e^{i \theta_{0}}$ and

$$
l(c) \leq l(\gamma), \quad l_{\infty}(c) \leq l_{\infty}(\gamma) .
$$

Here, we have to modify $c$ so that the terminal points of both paths coincides. Put $\bar{b}:=\sup \left\{t \in[a, b] ;\left|\gamma_{\mathbb{C}}(t)\right|=D\right\}$. Then define a path $\hat{\gamma}$ by 
connecting $\left.c\right|_{[a, \bar{b}]}$ and $\left.\gamma\right|_{[\bar{b}, b]}$. Here, to connect $c(\bar{b})$ and $\gamma(\bar{b})$, we add the path $c_{\theta_{0}}:\left[0, \theta_{0}\right] \rightarrow \partial L(D)$ defined by $c_{\theta_{0}}(t)=e^{i t} \gamma(\bar{b})$. Then by (A6), we obtain $l\left(c_{\theta_{0}}\right) \leq \sqrt{C_{1}\left(1+u^{\kappa}\right)} \sqrt{D}$ and $l_{\infty}\left(c_{\theta_{0}}\right) \leq \sqrt{C_{1} u^{\kappa}} \sqrt{D}$ if $|\gamma(\bar{b})| \leq u \leq R$. Hence we have the following proposition.

Proposition 7.5. Let $D \leq 1$ and $1 \leq u \leq R$, and $x, y, \gamma, \hat{\gamma}$ be as above. If the image of $\gamma$ is contained in $\mathbf{B}(u)$, then we have

$$
\begin{aligned}
l(\hat{\gamma})-l(\gamma) & \leq \sqrt{C_{1}\left(1+u^{\kappa}\right)} \sqrt{D} \\
l_{\infty}(\hat{\gamma})-l_{\infty}(\gamma) & \leq \sqrt{C_{1} u^{\kappa}} \sqrt{D} .
\end{aligned}
$$

Proposition 7.6. Let $x, y, \gamma, \hat{\gamma}$ be as above. If the image of $\gamma$ is contained in $\mathbf{B}(u)$, then the image of $\hat{\gamma}$ is contained in $\mathbf{B}(u+D) \backslash L(D)$.

Proof. It is obvious by the construction that the image of $\hat{\gamma}$ is contained in $\mathbb{R}^{3} \backslash L(D)$.

Since $S^{1}$-action preserves $\mathbf{B}(u)$ and

$$
\left|P_{\gamma}\right|^{2} \leq|\gamma|^{2}+D^{2}, \quad\left|\left(\gamma_{\mathbb{R}}(t), \frac{D \gamma_{\mathbb{C}}(t)}{\left|\gamma_{\mathbb{C}}(t)\right|}\right)\right|^{2} \leq|\gamma|^{2}+D^{2}
$$

holds, we have th assertion.

\subsection{Estimates (3)}

Let

$$
\begin{aligned}
\operatorname{Path}(u, D, x, y) & :=\{\gamma \in \operatorname{Path}(x, y) ; \operatorname{Im}(\gamma) \subset K(u, D)\} \\
d_{u, D}(x, y) & :=\inf _{\gamma \in \operatorname{Path}(u, D, x, y)} l(\gamma) \\
d_{\infty, u, D}(x, y) & :=\inf _{\gamma \in \operatorname{Path}(u, D, x, y)} l_{\infty}(\gamma) .
\end{aligned}
$$

for $x, y \in K(u, D)$. By the definition, $d(x, y) \leq d_{u, D}(x, y)$ always holds. In this subsection we consider the opposite estimate.

Lemma 7.7. Let $\hat{\zeta}:=\left(\zeta_{\mathbb{R}}, \frac{D \zeta_{\mathbb{C}}}{\mid \zeta_{\mathbb{C}}}\right)$ if $\zeta_{\mathbb{C}} \neq 0$, and $\hat{\zeta}:=\left(\zeta_{\mathbb{R}}, D\right)$ if $\zeta_{\mathbb{C}}=0$. Suppose $\Phi, \Phi_{\infty}$ satisfy (A3-6), and $1 \leq u \leq R$.

(1) If $\zeta \in L(D) \cap \mathbf{B}(u-1)$ and $0<D \leq 1$, then

$$
d_{u}(\zeta, \hat{\zeta}) \leq 2 \sqrt{C_{1}\left(1+u^{\kappa}\right) D}, \quad d_{\infty, u}(\zeta, \hat{\zeta}) \leq 2 \sqrt{C_{1} u^{\kappa} D}
$$

hold. 
(2) If $\zeta \in L(D) \cap K(u-1, D)$ and $0<D \leq 1$, then

$$
d_{u, D}(\zeta, \hat{\zeta}) \leq 2 \sqrt{C_{1}\left(1+u^{\kappa}\right) D}, \quad d_{\infty, u, D}(\zeta, \hat{\zeta}) \leq 2 \sqrt{C_{1} u^{\kappa} D}
$$

hold.

Proof. Let $\gamma(t)=\left(\zeta_{\mathbb{R}}, t \hat{\zeta}_{\mathbb{C}}\right)$ for $t \in\left[\left|\zeta_{\mathbb{C}}\right| / D, 1\right]$. Then $\gamma$ is joining $\zeta$ and $\hat{\zeta}$, and the image of $\gamma$ is contained in $\mathbf{B}(u-1+D) \subset \mathbf{B}(u)$. Then by $(\mathbf{A} 3,6)$ we have $\Phi(\gamma(t)) \leq C_{1}\left(1+u^{\kappa}\right) /(t D)$. Then we have

$$
d_{u}(\zeta, \hat{\zeta}) \leq l(\gamma) \leq 2 \sqrt{C_{1}\left(1+u^{\kappa}\right) D}
$$

Moreover, if $\zeta \in K(u-1, D)$, then the image of $\gamma$ is contained in $K(u, D)$, therefore we have

$$
d_{u, D}(\zeta, \hat{\zeta}) \leq l(\gamma) \leq 2 \sqrt{C_{1}\left(1+u^{\kappa}\right) D}
$$

The estimates for $d_{\infty, u}(\zeta, \hat{\zeta})$ and $d_{\infty, u, D}(\zeta, \hat{\zeta})$ follows in the same way.

Proposition 7.8. Suppose $\Phi, \Phi_{\infty}$ satisfy (A3-6) and let $\rho$ be as in Proposition 7.3. If $\rho(u+1)+1 \leq R$, then

$$
\begin{aligned}
\left|d_{\rho(u+1)+1, D}(x, y)-d(x, y)\right| & \leq \xi(u) \sqrt{D} \\
\left|d_{\infty, \rho(u+1)+1, D}(x, y)-d_{\infty}(x, y)\right| & \leq \xi_{\infty}(u) \sqrt{D}
\end{aligned}
$$

holds for any $x, y \in K(u, D)$ and $0<D \leq 1$, where

$$
\begin{aligned}
\xi(u) & :=\sqrt{C_{1}\left(1+(\rho(u+1)+1)^{\kappa}\right)}+8 \sqrt{C_{1}\left(1+(u+1)^{\kappa}\right)}+2, \\
\xi_{\infty}(u) & :=\sqrt{C_{1}(\rho(u+1)+1)^{\kappa}}+8 \sqrt{C_{1}(u+1)^{\kappa}}+2 .
\end{aligned}
$$

Proof. Since $d(x, y) \leq d_{\rho(u+1)+1, D}(x, y)$ always holds, it suffices to show that $d_{\rho(u+1)+1, D}(x, y)-d(x, y) \leq \xi(u) \sqrt{D}$. Let $x, y \in K(u, D)$ and $0<D \leq 1$. By the assumption $\rho(u+1)+1 \leq R$ and the definition of $\rho, u+1 \leq R$ holds. Define $\hat{x} \in \mathbb{R}^{3}$ as in Lemma 7.7 if $x \in L(D)$, and $\hat{x}:=x$ if $x \notin L(D)$. Define $\hat{y}$ in the same way. Then we can see $\hat{x}, \hat{y} \in \mathbf{B}(u+1) \backslash L(D)$ and $d_{u+1, D}(x, \hat{x}) \leq 2 \sqrt{C_{1}\left(1+(u+1)^{\kappa}\right) D}$ by Lemma 7.7, consequently we obtain

$$
d_{u+1, D}(x, \hat{x})+d_{u+1, D}(y, \hat{y}) \leq 4 \sqrt{C_{1}\left(1+(u+1)^{\kappa}\right) D} .
$$

For any $\gamma \in \operatorname{Path}(\hat{x}, \hat{y})$, we construct $F(\gamma) \in \operatorname{Path}(\rho(u+1)+1, D, \hat{x}, \hat{y})$ as follows. By the Proposition [7.3, we can see

$$
l(\gamma) \geq d(\hat{x}, \hat{y})=d_{\rho(u+1)}(\hat{x}, \hat{y})=\inf _{c \in \operatorname{Path}(\rho(u+1), x, y)} l(c),
$$


accordingly we can take $c \in \operatorname{Path}(\rho(u+1), x, y)$ such that $l(c) \leq l(\gamma)+\sqrt{D}$. Then we can apply the argument in Section 7.2 to $\hat{x}, \hat{y}$ and $c$ so that we obtain piecewise smooth paths $\hat{c}$ whose image is contained in $\mathbf{B}(\rho(u+1)+1) \backslash L(D)$, hence in $K(\rho(u+1)+1, D)$. Then we have

$$
\liminf _{k \rightarrow \infty} l(\hat{c})-l(c) \leq \sqrt{C_{1}\left(1+(\rho(u+1)+1)^{\kappa}\right) D},
$$

by Proposition [7.5. Therefore, there is a sufficiently large $k$, which may depend on $n$ and $D$, such that $l(\hat{c})-l(c) \leq \sqrt{C_{1}\left(1+(\rho(u+1)+1)^{\kappa}\right) D}+\sqrt{D}$. Put $F(\gamma)=\hat{c}$. Then we can see

$$
\begin{aligned}
l(F(\gamma))-l(\gamma) & \leq l(F(\gamma))-l(c)+l(c)-l(\gamma) \\
& \leq \sqrt{C_{1}\left(1+(\rho(u+1)+1)^{\kappa}\right) D}+\sqrt{D}+\sqrt{D} \\
& =\left\{\sqrt{C_{1}\left(1+(\rho(u+1)+1)^{\kappa}\right)}+2\right\} \sqrt{D} .
\end{aligned}
$$

Thus we obtain $F(\gamma) \in \operatorname{Path}(\rho(u+1)+1, D, \hat{x}, \hat{y})$ for every $\gamma \in \operatorname{Path}(\hat{x}, \hat{y})$, such that

$$
l(F(\gamma))-l(\gamma) \leq\left\{\sqrt{C_{1}\left(1+(\rho(u+1)+1)^{\kappa}\right)}+2\right\} \sqrt{D} .
$$

By taking the infimum of (11) for all $\gamma \in \operatorname{Path}(\hat{x}, \hat{y})$, we obtain

$$
d_{\rho(u+1)+1, D}(\hat{x}, \hat{y}) \leq d(\hat{x}, \hat{y})+\left\{\sqrt{C_{1}\left(1+(\rho(u+1)+1)^{\kappa}\right)}+2\right\} \sqrt{D} .
$$

Since $\rho(u+1) \geq u+1$, we have

$$
\begin{aligned}
\left|d_{\rho(u+1)+1, D}(\hat{x}, \hat{y})-d_{\rho(u+1)+1, D}(x, y)\right| & \leq d_{\rho(u+1)+1, D}(\hat{x}, x)+d_{\rho(u+1)+1, D}(\hat{y}, y) \\
& \leq d_{u+1, D}(\hat{x}, x)+d_{u+1, D}(\hat{y}, y) \\
& \leq 4 \sqrt{C_{1}\left(1+(u+1)^{\kappa}\right) D} \\
|d(\hat{x}, \hat{y})-d(x, y)| & \leq d(\hat{x}, x)+d(\hat{y}, y) \\
& \leq d_{u+1, D}(\hat{x}, x)+d_{u+1, D}(\hat{y}, y) \\
& \leq 4 \sqrt{C_{1}\left(1+(u+1)^{\kappa}\right) D}
\end{aligned}
$$

by (10), hence

$$
\begin{aligned}
d_{\rho(u+1)+1, D}(x, y) & \leq d_{\rho(u+1)+1, D}(\hat{x}, \hat{y})+4 \sqrt{C_{1}\left(1+(u+1)^{\kappa}\right) D} \\
d(\hat{x}, \hat{y}) & \leq d(x, y)+4 \sqrt{C_{1}\left(1+(u+1)^{\kappa}\right) D}
\end{aligned}
$$

hold. By combining these inequalities with (12), we obtain

$$
d_{\rho(u+1)+1, D}(x, y) \leq d(x, y)+\xi(u) \sqrt{D}
$$

The second inequality can be shown in the same way. 


\subsection{From (A3-6) to (A1-2)}

Proposition 7.9. Suppose that $\Phi, \Phi_{\infty}$ satisfy (A3-6), and let $\gamma:[a, b] \rightarrow$ $K(u, D)$ and $1 \leq u \leq R$. Then

$$
\left|l(\gamma)-l_{\infty}(\gamma)\right| \leq \sqrt{\frac{\varepsilon u}{C_{0} D^{m}}} l_{\infty}(\gamma)
$$

holds.

Proof. Since $l(\gamma)=\int_{a}^{b} \sqrt{\Phi(\gamma(t))}\left|\gamma^{\prime}(t)\right| d t$, one can see

$$
\begin{aligned}
\left|l(\gamma)-l_{\infty}(\gamma)\right| & \leq \int_{a}^{b} \sqrt{\left|\Phi(\gamma)-\Phi_{\infty}(\gamma)\right|} \gamma^{\prime} \mid d t \\
& \leq \int_{a}^{b} \sqrt{\frac{\left|\Phi(\gamma)-\Phi_{\infty}(\gamma)\right|}{\Phi_{\infty}(\gamma)}} \sqrt{\Phi_{\infty}(\gamma)}\left|\gamma^{\prime}\right| d t \\
& \leq \int_{a}^{b} \sqrt{\frac{\varepsilon \max \{|\gamma|, 1\}}{C_{0} D^{m}}} \sqrt{\Phi_{\infty}(\gamma(t))}\left|\gamma^{\prime}(t)\right| d t
\end{aligned}
$$

by $(\mathbf{A} 3, \mathbf{5})$. Since we have assumed $|\gamma| \leq u$ and $u \geq 1$, we have

$$
\left|l(\gamma)-l_{\infty}(\gamma)\right| \leq \sqrt{\frac{\varepsilon u}{C_{0} D^{m}}} l_{\infty}(\gamma)
$$

Proposition 7.10. Suppose that $\Phi, \Phi_{\infty}$ satisfy $(\mathbf{A 3}, 5,6)$, then,

$$
\left|d_{u, D}(x, y)-d_{\infty, u, D}(x, y)\right| \leq \sqrt{\frac{\varepsilon u}{C_{0} D^{m}}} d_{\infty, u, D}(x, y)
$$

holds for all $1 \leq u \leq R$.

Proof. Put $\delta=\sqrt{\frac{\varepsilon u}{C_{0} D^{m}}}$. Then Proposition 7.9 gives

$$
(1-\delta) l_{\infty}(\gamma) \leq l(\gamma) \leq(1+\delta) l_{\infty}(\gamma)
$$

Then by taking the infimum of (13) for all $\gamma \in \operatorname{Path}(u, D, x, y)$, we can see

$$
(1-\delta) d_{\infty, u, D}(x, y) \leq d_{n, u, D}(x, y) \leq(1+\delta) d_{\infty, u, D}(x, y)
$$

for all $u \geq 0$. 
Proposition 7.11. Suppose that $\Phi, \Phi_{\infty}$ satisfy (A3-6) $u \leq 1$, and let $u^{(2)}:=$ $\rho(u+2)+1 \leq R$. Then we have

$$
\begin{array}{rl}
\left|d(x, y)-d_{\infty}(x, y)\right| \leq 2 & 6 \sqrt{C_{1}\left(1+R^{\kappa}\right) D}+4 \sqrt{D} \\
& +\sqrt{\frac{\varepsilon R}{C_{0} D^{m}}}\left\{C_{2} R^{\kappa^{\prime}}+\left(9 \sqrt{C_{1} R^{\kappa}}+2\right) \sqrt{D}\right\} .
\end{array}
$$

for all $x, y \in \mathbf{B}(u)$.

Proof. Put $u^{(1)}=\rho(u+1)+1$ and let $x, y \in K(u, D)$. Then $u^{(1)} \leq R$. By combining Propositions 7.8 and 7.10 , we have

$$
\begin{aligned}
\left|d(x, y)-d_{\infty}(x, y)\right| \leq \mid & d(x, y)-d_{u^{(1)}, D}(x, y)|+| d_{u^{(1)}, D}(x, y)-d_{\infty, u^{(1)}, D}(x, y) \mid \\
& +\left|d_{\infty}(x, y)-d_{\infty, u^{(1)}, D}(x, y)\right| \\
\leq & \xi(u) \sqrt{D}+\xi_{\infty}(u) \sqrt{D}+\sqrt{\frac{\varepsilon u^{(1)}}{C_{0} D^{m}}} d_{\infty, u^{(1)}, D}(x, y) \\
\leq & 2 \xi(u) \sqrt{D}+\sqrt{\frac{\varepsilon u^{(1)}}{C_{0} D^{m}}}\left(d_{\infty}(x, y)+\xi_{\infty}(u) \sqrt{D}\right) .
\end{aligned}
$$

By the Proposition [7.2, $D_{\infty, u}<C_{2} u^{\kappa^{\prime}}$ holds if $u \geq 1$, consequently $d_{\infty}(x, y)$ is not more than $C_{2} u^{\kappa^{\prime}}$. Therefore we obtain

$$
\left|d(x, y)-d_{\infty}(x, y)\right| \leq 2 \xi(u) \sqrt{D}+\sqrt{\frac{\varepsilon u^{(1)}}{C_{0} D^{m}}}\left(C_{2} u^{\kappa^{\prime}}+\xi_{\infty}(u) \sqrt{D}\right)
$$

for all $x, y \in K(u, D)$.

Next we consider the case of $x \in \mathbf{B}(u)$ but not contained in $K(u, D)$. In this case $x \in \mathbf{B}(u) \cap L(D)$ holds, hence we can apply Lemma 7.7. Let $\hat{x}$ be as in Lemma 7.7. Then we can see that

$$
d(x, \hat{x}) \leq 2 \sqrt{C_{1}\left(1+(u+1)^{\kappa}\right) D}
$$

and $\hat{x}$ is contained in $K(u+1, D)$. Here we suppose that $y$ is also contained in $\mathbf{B}(u) \cap L(D)$, and follow the same procedure. If $y$ is in $K(u, D)$, then suppose $y=\hat{y}$ in the following discussion. Now we have

$$
|d(x, y)-d(\hat{x}, \hat{y})| \leq d(x, \hat{x})+d(y, \hat{y}) \leq 4 \sqrt{C_{1}\left(1+(u+1)^{\kappa}\right) D},
$$

hence we can see

$$
\begin{aligned}
\left|d(x, y)-d_{\infty}(x, y)\right| \leq & 8 \sqrt{C_{1}\left(1+(u+1)^{\kappa}\right) D}+\left|d(\hat{x}, \hat{y})-d_{\infty}(\hat{x}, \hat{y})\right| \\
\leq & 8 \sqrt{C_{1}\left(1+(u+1)^{\kappa}\right) D}+2 \xi(u+1) \sqrt{D} \\
& \quad+\sqrt{\frac{\varepsilon u^{(2)}}{C_{0} D^{m}}}\left\{C_{2}(u+1)^{\kappa^{\prime}}+\xi_{\infty}(u+1) \sqrt{D}\right\} .
\end{aligned}
$$


Since $\xi(u)$ is monotonically increasing and $u+2 \leq u^{(2)} \leq R$ holds, we have

$$
\xi(u+1) \leq 9 \sqrt{C_{1}\left(1+R^{\kappa}\right)}+2, \quad \xi_{\infty}(u+1) \leq 9 \sqrt{C_{1} R^{\kappa}}+2
$$

Corollary 7.12. Suppose that $\Phi, \Phi_{\infty}$ satisfy (A3-6) and $\varepsilon \leq 1$, and let $u^{(2)}:=\rho(u+2)+1 \leq R$. Then there exists a constant $C$ independent of any other constants such that

$$
\left|d(x, y)-d_{\infty}(x, y)\right|<C\left(1+\sqrt{C_{1}}\right)\left(1+C_{0}^{-\frac{1}{2}}\right) R^{1+\frac{\kappa}{2}} \varepsilon^{\frac{1}{2(1+m)}} .
$$

for all $x, y \in \mathbf{B}(u)$.

Proof. In Proposition [7.11, let $D=\varepsilon^{\frac{1}{1+m}} \leq 1$. As described in the proof of Proposition 7.2, $C_{2}$ is linearly depending on $\sqrt{C_{1}}$. Then assertion follows by using $R \geq 1, \varepsilon \leq 1$ and unifying constants.

Proposition 7.13. Suppose that $\Phi(\zeta) \geq \frac{A}{|\zeta|}$ holds for some $A>0$ and all $\zeta$ with $|\zeta| \leq 1$, and let $u(r):=\left(1+\frac{A^{-\frac{1}{2}} r}{2}\right)^{2}$. Then $B(0, r) \subset \mathbf{B}(u(r))$ holds for all $r>0$, where $B(0, r)$ are the metric ball with respect to $d$.

Proof. Let $\zeta \in B(0, r)$. Then by the same argument in the proof of the first inequality of Proposition 7.2 we have

$$
2 \sqrt{A}(\sqrt{|\zeta|}-1) \leq d(0, \zeta)<r
$$

which gives $|\zeta|<\left(1+\frac{A^{-\frac{1}{2}} r}{2}\right)^{2}=u(r)$.

Proposition 7.14. Suppose that $\Phi, \Phi_{\infty}$ satisfy (A3-6) and suppose $\varepsilon \leq 1$. Then the identity map of $\mathbb{R}^{3}$ is $(r, \delta)$-isometry from $\left(\mathbb{R}^{3}, d, 0\right)$ to $\left(\mathbb{R}^{3}, d_{\infty}, 0\right)$, where $r, \delta>0$ are defined by

$$
\rho(u(r)+2)+1=R, \quad \delta=C\left(1+\sqrt{C_{1}}\right)\left(1+C_{0}^{-\frac{1}{2}}\right) R^{1+\frac{\kappa}{2}} \varepsilon^{\frac{1}{2(1+m)}} .
$$

Proof. Let $x, y \in B(0, r)$. Then $x, y \in \mathbf{B}(u(r))$, hence

$$
\left|d(x, y)-d_{\infty}(x, y)\right|<C\left(1+\sqrt{C_{1}}\right)\left(1+C_{0}^{-\frac{1}{2}}\right) R^{1+\frac{\kappa}{2}} \varepsilon^{\frac{1}{2(1+m)}}
$$

holds. Next we show $B_{\infty}(0, r-\delta) \subset B(B(0, r), \delta)$. If $x \in B_{\infty}(0, r-\delta)$, then $x \in \mathbf{B}(u(r))$ holds, therefore (14) gives

$$
\begin{aligned}
d(0, x) & <d_{\infty}(0, x)+C\left(1+\sqrt{C_{1}}\right)\left(1+C_{0}^{-\frac{1}{2}}\right) R^{1+\frac{\kappa}{2}} \varepsilon^{\frac{1}{2(1+m)}} \\
& <r-\delta+C\left(1+\sqrt{C_{1}}\right)\left(1+C_{0}^{-\frac{1}{2}}\right) R^{1+\frac{\kappa}{2}} \varepsilon^{\frac{1}{2(1+m)}}=r
\end{aligned}
$$

which implies $B_{\infty}(0, r-\delta) \subset B(0, r)$. 
By Propositions 7.13, and Proposition 6.3, the following estimate is obtained.

Proposition 7.15. Let $\Phi_{a}$ be as in Section [6] and assume $\sum_{n=0}^{\infty} A_{S_{n}, P}^{T_{n}}-$ $2 a^{\frac{1}{1+\alpha}}>0$. Then $\sup _{\zeta \in B(0, r)} \frac{1}{N \sqrt{\Phi_{a}(\zeta)}}$ is not more than

$$
\frac{\left(\frac{a}{P}\right)^{\frac{1}{1+\alpha}}}{\sqrt{\sum_{n=0}^{\infty} A_{S_{n}, P}^{T_{n}}-2\left(\frac{a}{P}\right)^{\frac{1}{1+\alpha}}}}\left(1+\frac{r}{2 \sqrt{\sum_{n=0}^{\infty} A_{S_{n}, P}^{T_{n}}-2\left(\frac{a}{P}\right)^{\frac{1}{1+\alpha}}}}\right) .
$$

Combining Propositions 7.14 and 7.15, we obtain the following theorem.

Theorem 7.16. Let $a_{i}, P_{i}, n_{i}>0, \lim _{i \rightarrow \infty} a_{i}=0$ and $\lim _{i \rightarrow \infty} n_{i} \rightarrow \infty$. Put $S_{i, n_{i}}, T_{i, n_{i}}$ as in Section [6. Suppose that there are constants $\varepsilon=\varepsilon_{i}(R), C_{0}$, $C_{1}, \kappa, m$ for all $R \geq 1$ such that $\Phi=\Phi_{a_{i}}$ and $\Phi_{\infty}$ satisfy (A3-6). If

$$
\lim _{i \rightarrow \infty} \varepsilon_{i}(R)=\lim _{i \rightarrow \infty} \frac{a_{i}}{P_{i}}=0, \quad \liminf _{i \rightarrow \infty} \sum_{l=0}^{\infty} A_{S_{i, n_{i}}, P_{i}}^{T_{i, n_{i}}}>0
$$

and $C_{0}, C_{1}, \kappa, m$ are independent of $i, R$, then

$$
\left\{\left(X, a_{i} g_{\Lambda}, p\right)\right\}_{i} \underset{i \rightarrow \infty}{\stackrel{G H}{\longrightarrow}}\left(\mathbb{R}^{3}, d_{\infty}, 0\right) .
$$

Proof. Fix $r>0$ and $\delta>0$ arbitrarily. Put $R(r)=\rho(u(r)+2)+1$, and let $C>0$ be the constant in Corollary 7.12, By the assumption, there exists $i(r, \delta)>0$ such that $C\left(1+\sqrt{C_{1}}\right)\left(1+C_{0}^{-\frac{1}{2}}\right) R(r)^{1+\frac{\kappa}{2}} \varepsilon_{i}(R(r))^{\frac{1}{2(1+m)}}<\frac{\delta}{2}$ holds for all $i \geq i(r, \delta)$. Then by Proposition $7.14, \mathrm{id}_{\mathbb{R}^{3}}$ is the $\left(r, \frac{\delta}{2}\right)$-isometry from $\left(\mathbb{R}^{3}, d_{a_{i}}, 0\right)$ to $\left(\mathbb{R}^{3}, d_{a_{\infty}}, 0\right)$. By Proposition 7.15 , we can take $i^{\prime}(r, \delta) \geq i(r, \delta)$ such that $\sup _{\zeta \in B(0, r)} \frac{1}{N \sqrt{\Phi_{a}(\zeta)}}<\frac{\delta}{2}$ for all $i \geq i^{\prime}(r, \delta)$. Then Proposition 5.1 gives the assertion.

\section{Convergence}

In this section we consider the convergence of $\left\{\left(X, a_{i} g_{\Lambda}\right)\right\}_{i}$, where $\Lambda$ is the one defined in Section 6, and $\left\{a_{i}\right\}_{i}$ is a sequence with $a_{i}>0$ and $\lim _{i \rightarrow \infty} a_{i}$, applying Theorem 7.16. To apply them, we have to estimate constants $\varepsilon, C_{0}, C_{1}$ in (A3-6) uniformly with respect to $i \in \mathbb{N}$, and show that $\varepsilon \rightarrow 0$ as $i \rightarrow \infty$. In Section 8.1, we consider the uniform estimate for the case of $P=1$, which is the simplest case. In Sections 8.2 and 8.3, we suppose $P$ is depending on some parameters. Then we apply them to show Theorems 1.2 and 1.3 in Sections 8.4 and 8.5 . 
Put $S_{a, n}:=a^{\frac{1}{1+\alpha}} K_{2 n}$ and $T_{a, n}:=a^{\frac{1}{1+\alpha}} K_{2 n+1}$. We take a subsequence

$$
\left\{K_{n_{0}}<K_{n_{1}}<K_{n_{2}}<\cdots\right\} \subset\left\{K_{0}<K_{1}<K_{2}<\cdots\right\} .
$$

We are now going to consider the convergence in several cases according to the rate of the convergence of $\left\{a_{i}\right\}_{i}$, or the divergence of $\left\{K_{n}\right\}_{n}$.

From now on, we put

$$
\begin{aligned}
\Phi_{S}^{T}(\zeta) & :=\Phi_{S, 1}^{T}(\zeta)=\int_{S}^{T} \frac{d x}{\left|\zeta-\left(x^{\alpha}, 0,0\right)\right|}, \\
A_{S}^{T} & :=A_{S, 1}^{T}=\int_{S}^{T} \frac{d x}{1+x^{\alpha}} .
\end{aligned}
$$

\subsection{Convergence (1)}

Fix $a>0, n, 0 \leq S<T \leq \infty$ and put $P=1$.

Proposition 8.1. Let $R \geq 1$ and $D \leq 1$. There exists a constant $C_{\alpha}>0$ depending only on $\alpha$ such that

$$
\begin{aligned}
\left|\Phi_{a}(\zeta)-\Phi_{S}^{T}(\zeta)\right| \leq & \frac{C_{\alpha} \varepsilon_{n}}{D} \\
\varepsilon_{a, n}= & a^{\frac{1}{1+\alpha}}+\frac{K_{2 n-1}}{K_{2 n}} S_{a, n}+\left(\frac{K_{2 n+2}}{K_{2 n+1}} T_{a, n}\right)^{-\alpha+1} \\
& \quad+\left|S_{a, n}-S\right|+\left|T_{a, n}^{-\alpha+1}-T^{-\alpha+1}\right|
\end{aligned}
$$

holds for any $\zeta \in K(R, D)$.

Proof. By combining Propositions 6.1 and (7) (18), we have

$$
\left|\Phi_{a}(\zeta)-\Phi_{S_{a, n}}^{T_{a, n}}(\zeta)\right| \leq \frac{2 a^{\frac{1}{1+\alpha}}}{D}+\frac{T_{a, n-1}}{D}+\frac{2 S_{a, n+1}^{-\alpha+1}}{\alpha-1}
$$

if $S_{a, n+1} \geq(2|\zeta|)^{\frac{1}{\alpha}}$. Here, $|\zeta| \leq R$ and

$$
\begin{aligned}
& S_{a, n+1}=a^{\frac{1}{1+\alpha}} K_{2 n+2}=\frac{K_{2 n+2}}{K_{2 n+1}} T_{a, n}, \\
& T_{a, n-1}=a^{\frac{1}{1+\alpha}} K_{2 n-1}=\frac{K_{2 n-1}}{K_{2 n}} S_{a, n} .
\end{aligned}
$$

On the other hand, we can see

$$
\left|\Phi_{S_{a, n}}^{T_{a, n}}(\zeta)-\Phi_{S}^{T}(\zeta)\right| \leq \frac{\left|S_{a, n}-S\right|}{D}+\frac{2\left|T_{a, n}^{-\alpha+1}-T^{-\alpha+1}\right|}{\alpha-1},
$$

we obtain the assertion. 
Now, we put $\Phi=\Phi_{a}, \Phi_{\infty}=\Phi_{S}^{T}$, and suppose $a,\left|S_{a, n}-S\right|$ and $\mid T_{a, n}^{-\alpha+1}-$ $T^{-\alpha+1} \mid$ are sufficiently small. Then the constants in (A3-6) can be taken uniformly such as

$$
C_{0}=\frac{1}{2} A_{S}^{T}, \quad C_{1}=\frac{\alpha 2^{\frac{1}{\alpha}}}{\alpha-1}, \quad m=1, \quad \kappa=\frac{1}{\alpha} .
$$

Then by Proposition [8.1, if $\lim _{n \rightarrow \infty} \frac{K_{2 n+1}}{2 n}=\infty$, then we have $\varepsilon_{a, n} \rightarrow 0$ as $a \rightarrow 0, n \rightarrow \infty,\left|S_{a, n}-S\right| \rightarrow 0$ and $\left|T_{a, n}^{-\alpha+1}-T^{-\alpha+1}\right| \rightarrow 0$. Hence by Theorem 7.16, we have the next results.

Theorem 8.2. Let $\left(X, g_{\Lambda}\right)$ be as in Section 6 and suppose $\lim _{n \rightarrow \infty} \frac{K_{2 n+1}}{K_{2 n}}=$ $\infty$. Assume that $\left\{a_{i}\right\}_{i} \subset \mathbb{R}^{+}$and

$$
\left\{K_{n_{0}}<K_{n_{1}}<K_{n_{2}}<\cdots\right\} \subset\left\{K_{0}<K_{1}<K_{2}<\cdots\right\} .
$$

satisfies

$$
\lim _{i \rightarrow \infty} a_{i}^{\frac{1}{1+\alpha}} K_{2 n_{i}}=S \geq 0, \quad \lim _{i \rightarrow \infty} a_{i}^{\frac{1}{1+\alpha}} K_{2 n_{i}+1}=T \leq \infty, \quad S<T .
$$

Then $\left\{\left(X, a_{n} g_{\Lambda}, p\right)\right\}_{n} \stackrel{G H}{\longrightarrow}\left(\mathbb{R}^{3}, d_{S}^{T}, 0\right)$, where $d_{S}^{T}$ is the metric induced by $\Phi_{S}^{T}$. $h_{0}$.

\subsection{Convergence (2)}

Let $\left(X, d_{X}, p\right)$ and $\left(Y, d_{Y}, q\right)$ be pointed metric spaces and $\lim _{n \rightarrow \infty} a_{n}=0$. Assume that $\left\{\left(X, a_{n} d_{X}, p\right)\right\}_{n} \stackrel{G H}{\longrightarrow}\left(Y, d_{Y}, q\right)$. Then it is easy to check that $\left\{\left(X, s a_{n} d_{X}, p\right)\right\}_{n} \stackrel{G H}{\longrightarrow}\left(Y, s d_{Y}, q\right)$ for any $s>0$. Moreover, if $\left\{a_{n, m}\right\}_{n, m \in \mathbb{N}}$ satisfies $\lim _{n \rightarrow \infty} a_{n, m}=0$ for every $m$ and

$$
\left\{\left(X, a_{n, m} d_{X}, p\right)\right\}_{n} \stackrel{G H}{\longrightarrow}\left(Y_{m}, d_{Y_{m}}, q_{m}\right), \quad\left\{\left(Y_{m}, d_{Y_{m}}, q_{m}\right)\right\}_{m} \stackrel{G H}{\longrightarrow}\left(Y, d_{Y}, q\right),
$$

hold for every $m$, then by the diagonal argument one can show there exists a subset $\left\{a_{n, m(n)}\right\}_{n} \subset\left\{a_{n, m}\right\}_{n, m}$ such that $\lim _{n \rightarrow \infty} a_{n, m(n)}=0$ and

$$
\left\{\left(X, a_{n, m(n)} d_{X}, p\right)\right\}_{n} \stackrel{G H}{\longrightarrow}\left(Y, d_{Y}, q\right) .
$$

Now, let $\mathcal{T}(X, d)$ be the set of isometry classes of tangent cones at infinity of $(X, d)$. From the above argument, one can see that $\mathcal{T}(X, d)$ is closed with respect to the pointed Gromov-Hausdorff topology, and if $\left(Y, d^{\prime}\right) \in \mathcal{T}(X, d)$, then its rescaling $\left(Y, a d^{\prime}\right)$ is also contained in $\mathcal{T}(X, d)$. 
From Section $8.1,\left(\mathbb{R}^{3}, d_{S}^{T}, 0\right)$ may appear as the tangent cone at infinity of some $\left(X, g_{\Lambda}\right)$, where $\Lambda$ is as in Section 6 .

Let $\sigma>0,0 \leq S<T \leq \infty$ and $I_{\sigma}: \zeta \mapsto \sigma^{-1} \zeta$ be the dilation. Then we have

$$
I_{P^{\frac{1}{1+\alpha}}}^{*}\left(\Phi_{S}^{T} h_{0}\right)=P^{\frac{-1}{\alpha}} \Phi_{P^{\frac{1}{\alpha(1+\alpha)}} S}^{\frac{1}{\alpha(1+\alpha)}}(\zeta) h_{0}=\Phi_{S^{\prime}, P}^{T^{\prime}}(\zeta) h_{0},
$$

where

$$
S^{\prime}=P^{\frac{-1}{1+\alpha}} S, \quad T^{\prime}=P^{\frac{-1}{1+\alpha}} T .
$$

Hence if $\left(\mathbb{R}^{3}, d_{S}^{T}, 0\right) \in \mathcal{T}\left(X, g_{\Lambda}\right)$, then $\left\{\left(\mathbb{R}^{3}, d_{\sigma S}^{\sigma T}, 0\right)\right\}_{\sigma \in \mathbb{R}^{+}}$is also contained in $\mathcal{T}\left(X, g_{\Lambda}\right)$.

(1). Fix a constant $\theta>0$, put $P^{\frac{1}{1+\alpha}}=\theta \sqrt{S^{-\alpha+1}-T^{-\alpha+1}}>0$, and let $S^{\prime}, T^{\prime}$ be defined by (15).

Proposition 8.3. Let $R \geq 1$. There exists a constant $C>0$ depending only on $\alpha$ such that

$$
\left|\Phi_{S^{\prime}, P}^{T^{\prime}}(\zeta)-\frac{1}{\theta^{2}(\alpha-1)}\right| \leq \frac{C R}{\theta^{3} S^{\alpha} \sqrt{S^{-\alpha+1}-T^{-\alpha+1}}}
$$

holds for any $\zeta \in K(R, D)$ if $\theta S^{\alpha} \sqrt{S^{-\alpha+1}-T^{-\alpha+1}} \geq 2 R$.

Proof. Note that

$$
\Phi_{S^{\prime}, P}^{T^{\prime}}(\zeta)=P^{\frac{-1}{\alpha}} \int_{P^{\frac{1}{\alpha(1+\alpha)}} S}^{P^{\frac{1}{\alpha(1+\alpha)}} T} \frac{d x}{\left|\zeta-\left(x^{\alpha}, 0,0\right)\right|} .
$$

By the assumption, we have $P^{\frac{1}{1+\alpha}} S^{\alpha}=\theta S^{\alpha} \sqrt{S^{-\alpha+1}-T^{-\alpha+1}} \geq 2 R$, then we can see

$$
\begin{aligned}
& \left|\int_{P^{\frac{1}{\alpha(1+\alpha)}} S}^{P^{\frac{1}{\alpha(1+\alpha)}} T} \frac{d x}{\left|\zeta-\left(x^{\alpha}, 0,0\right)\right|}-\int_{P^{\frac{1}{\alpha(1+\alpha)}} S}^{P^{\frac{1}{\alpha(1+\alpha)}} T} \frac{d x}{x^{\alpha}}\right| \\
\leq & \int_{P^{\frac{1}{\alpha(1+\alpha)}} S}^{P^{\frac{1}{\alpha(1+\alpha)}} T}\left|\frac{1}{\left|\zeta-\left(x^{\alpha}, 0,0\right)\right|}-\frac{1}{x^{\alpha}}\right| d x \\
\leq & \int_{P^{\frac{1}{\alpha(1+\alpha)}} S}^{P^{\frac{1}{\alpha(1+\alpha)}} T} \frac{2 x^{\alpha}|\zeta|+|\zeta|^{2}}{\left|\zeta-\left(x^{\alpha}, 0,0\right)\right| x^{\alpha}\left(\left|\zeta-\left(x^{\alpha}, 0,0\right)\right|+x^{\alpha}\right)} d x \\
\leq & \int_{P^{\frac{1}{\alpha(1+\alpha)}} S}^{P^{\frac{1}{\alpha(1+\alpha)}} T} \frac{8 R}{x^{2 \alpha}} d x+\int_{P^{\frac{1}{\alpha(1+\alpha)}} S}^{P^{\frac{1}{\alpha(1+\alpha)}} T} \frac{4 R^{2}}{x^{3 \alpha}} d x \\
\leq & \frac{8 R P^{\frac{-2 \alpha+1}{\alpha(1+\alpha)}}}{2 \alpha-1}\left(S^{-2 \alpha+1}-T^{-2 \alpha+1}\right)+\frac{4 R^{2} P^{\frac{-3 \alpha+1}{\alpha(1+\alpha)}}}{3 \alpha-1}\left(S^{-3 \alpha+1}-T^{-3 \alpha+1}\right) .
\end{aligned}
$$


Since we have

$$
\int_{P^{\frac{1}{\alpha(1+\alpha)}}}^{P^{\frac{1}{\alpha(1+\alpha)}} T} \frac{d x}{x^{\alpha}}=\frac{P^{\frac{-\alpha+1}{\alpha(1+\alpha)}}}{\alpha-1}\left(S^{-\alpha+1}-T^{-\alpha+1}\right)=\frac{P^{\frac{1}{\alpha}}}{\theta^{2}(\alpha-1)},
$$

we obtain

$$
\begin{aligned}
\left|\Phi_{S^{\prime}, P}^{T^{\prime}}(\zeta)-\frac{1}{\theta^{2}(\alpha-1)}\right| \leq & \frac{8 R P^{\frac{-3}{1+\alpha}}}{2 \alpha-1}\left(S^{-2 \alpha+1}-T^{-2 \alpha+1}\right) \\
& +\frac{4 R^{2} P^{\frac{-4}{1+\alpha}}}{3 \alpha-1}\left(S^{-3 \alpha+1}-T^{-3 \alpha+1}\right) .
\end{aligned}
$$

Using the assumption $2 R \leq P^{\frac{1}{1+\alpha}} S^{\alpha}$ once more, we have

$$
\begin{aligned}
\left|\Phi_{S^{\prime}, P}^{T^{\prime}}(\zeta)-\frac{1}{\theta^{2}(\alpha-1)}\right| \leq & \frac{8 R P^{\frac{-3}{1+\alpha}}}{2 \alpha-1}\left(S^{-2 \alpha+1}-T^{-2 \alpha+1}\right) \\
& +\frac{2 R P^{\frac{-3}{1+\alpha}}}{3 \alpha-1}\left(S^{-2 \alpha+1}-S^{\alpha} T^{-3 \alpha+1}\right) \\
\leq & \theta^{-3} C_{\alpha} R S^{-\frac{1+\alpha}{2}} \frac{1-(S / T)^{3 \alpha-1}}{\left\{1-(S / T)^{\alpha-1}\right\}^{\frac{3}{2}}} .
\end{aligned}
$$

Now, put $f(x):=\frac{1-x^{3 \alpha-1}}{\left(1-x^{\alpha-1}\right)^{\frac{3}{2}}}$ for $0 \leq x<1$. Then there exists a constant $C_{\alpha}^{\prime}>$ 0 such that $f(x) \leq C_{\alpha}^{\prime}\left(1-x^{\alpha-1}\right)^{-\frac{1}{2}}$ holds for all $0 \leq x<1$. Consequently, by replacing $C_{\alpha}$ larger if necessary, we can see

$$
\left|\Phi_{S^{\prime}, P}^{T^{\prime}}(\zeta)-\frac{1}{\theta^{2}(\alpha-1)}\right| \leq \frac{C_{\alpha} R}{\theta^{3} S^{\alpha} \sqrt{S^{-\alpha+1}-T^{-\alpha+1}}} .
$$

Proposition 8.4. Suppose $\theta S^{\alpha} \sqrt{S^{-\alpha+1}-T^{-\alpha+1}} \geq 2 R$ for $R \geq 1$. Then

$$
A_{S^{\prime}, P}^{T^{\prime}} \geq \frac{1}{2 \theta^{2}(\alpha-1)}, \quad \Phi_{S^{\prime}, P}^{T^{\prime}}\left(\zeta_{\mathbb{R}}, \zeta_{\mathbb{C}}\right) \leq \frac{2|\zeta|}{\theta^{2}(\alpha-1)\left|\zeta_{\mathbb{C}}\right|}
$$

holds for any $\zeta=\left(\zeta_{\mathbb{R}}, \zeta_{\mathbb{C}}\right) \in \mathbb{R}^{3}=\mathbb{R} \oplus \mathbb{C}$ with $|\zeta| \leq R$.

Proof. We have $1 \leq S^{-\alpha} x^{\alpha}$ for all $x \geq S$, then we can see

$$
\begin{aligned}
A_{S^{\prime}, P}^{T^{\prime}} & \geq P^{-\frac{1}{1+\alpha}} \int_{S}^{T} \frac{d x}{S^{-\alpha} x^{\alpha}+P^{\frac{1}{1+\alpha}} x^{\alpha}} \\
& =\frac{1}{P^{\frac{1}{1+\alpha}}\left(S^{-\alpha}+P^{\frac{1}{1+\alpha}}\right)} \int_{S}^{T} \frac{d x}{x^{\alpha}} \\
& =\frac{1}{P^{\frac{1}{1+\alpha}} S^{-\alpha}\left(1+S^{\alpha} P^{\frac{1}{1+\alpha}}\right)} \frac{S^{-\alpha+1}-T^{-\alpha+1}}{\alpha-1} .
\end{aligned}
$$


Since we have

$$
S^{\alpha} P^{\frac{1}{1+\alpha}}=\theta \sqrt{S^{-\alpha+1}-T^{-\alpha+1}} \geq 2 R \geq 1
$$

we obtain

$$
A_{S^{\prime}, P}^{T^{\prime}} \geq \frac{S^{-\alpha+1}-T^{-\alpha+1}}{2(\alpha-1) P^{\frac{1}{1+\alpha}} S^{-\alpha} \cdot S^{\alpha} P^{\frac{1}{1+\alpha}}}=\frac{1}{2 \theta^{2}(\alpha-1)}
$$

Next we consider the upper estimate of $\Phi_{S^{\prime}, P}^{T^{\prime}}(\zeta)$. Take $\zeta$ such that $|\zeta| \leq$ $R$, then we have $2|\zeta| \leq P^{\frac{1}{1+\alpha}} S^{\alpha}$ by the assumption. Then one can see

$$
\begin{aligned}
\Phi_{S^{\prime}, P}^{T^{\prime}}(\zeta) \leq P^{-\frac{1}{1+\alpha}} \int_{S}^{T} \frac{2 d x}{P^{\frac{1}{1+\alpha}} x^{\alpha}} & =P^{\frac{-2}{1+\alpha}} \frac{2\left(S^{-\alpha+1}-T^{-\alpha+1}\right)}{\alpha-1} \\
& \leq \frac{2}{\theta^{2}(\alpha-1)} \leq \frac{2|\zeta|}{\theta^{2}(\alpha-1)\left|\zeta_{\mathbb{C}}\right|}
\end{aligned}
$$

Proposition 8.5. Let $\Phi=\Phi_{S^{\prime}, P}^{T^{\prime}}$ and $\Phi_{\infty} \equiv \frac{1}{\theta^{2}(\alpha-1)}$. Then there exists $C>0$ such that $\Phi, \Phi_{\infty}$ satisfy (A3-6) for $R \geq 1$ and

$$
\begin{aligned}
& m=1, \quad \varepsilon=\frac{C R}{\theta^{3} S^{\alpha} \sqrt{S^{-\alpha+1}-T^{-\alpha+1}}}, \quad C_{0}=\frac{1}{2 \theta^{2}(\alpha-1)}, \\
& C_{1}=\frac{1}{\theta^{2}} \max \left\{\frac{1}{\alpha-1}, \frac{C}{2}\right\}, \quad \kappa=1,
\end{aligned}
$$

if $\theta S^{\alpha} \sqrt{S^{-\alpha+1}-T^{-\alpha+1}} \geq 2 R$.

Proof. It is obvious that (A4) holds. Proposition 8.4 gives (A5) for $C_{0}=$ $\frac{1}{2 \theta^{2}(\alpha-1)}$ if we take $\theta S^{\alpha} \sqrt{S^{-\alpha+1}-T^{-\alpha+1}} \geq 2 R$. (A6) holds for $C_{1}=\frac{1}{\theta^{2}(\alpha-1)}$ since $\frac{1}{\alpha-1}=\frac{1}{\alpha-1} \frac{|\zeta|}{|\zeta|} \leq \frac{1}{\alpha-1} \frac{|\zeta|}{|\zeta \mathbb{C}|}$. Combining $\theta S^{\alpha} \sqrt{S^{-\alpha+1}-T^{-\alpha+1}} \geq 2 R$ and Proposition 8.3, we can see $\varepsilon \leq \frac{C}{2 \theta^{2}}$.

Now, Propositions 7.14 and 8.5 with $\theta=1$ gives the following theorem.

Theorem 8.6. Let $\left\{S_{i}\right\}_{i}$ and $\left\{T_{i}\right\}_{i}$ be sequences such that $0 \leq S_{i}<T_{i} \leq$ $\infty$ and $\lim _{i \rightarrow \infty} S_{i}^{\alpha} \sqrt{S_{i}^{-\alpha+1}-T_{i}^{-\alpha+1}}=\infty$, then $\left\{\left(\mathbb{R}^{3}, d_{S_{i}}^{T_{i}}, 0\right)\right\}_{i}$ converges to $\left(\mathbb{R}^{3}, h_{0}, 0\right)$ in the pointed Gromov-Hausdorff topology. 
(2). Next put $P^{\frac{1}{1+\alpha}}=\theta|T-S|$ for $0 \leq S<T$ and $\theta>0$, and let $S^{\prime}, T^{\prime}$ be as in (15). Then we can show the following similarly to Proposition 8.9.

Proposition 8.7. Let $D \geq 1$. We have

$$
\begin{aligned}
& \left|\Phi_{S^{\prime}, P}^{T^{\prime}}(\zeta)-\frac{1}{\theta|\zeta|}\right| \leq \frac{2}{\theta D}, \\
& \left|\Phi_{S^{\prime}, P}^{T^{\prime}}(\zeta)-\frac{1}{\theta|\zeta|}\right| \leq \frac{1+\theta T^{\alpha}(T-S)}{D^{3}} T^{\alpha}(T-S) .
\end{aligned}
$$

for all $\zeta \in K(R, D)$.

Proof. The first inequality is obviously shown by $\Phi_{S^{\prime}, P}^{T^{\prime}}(\zeta) \leq \frac{1}{\theta D}$ and $\frac{1}{|\zeta|} \leq \frac{1}{D}$. The second inequality follows from

$$
\begin{aligned}
& \left|\int_{P^{\frac{1}{\alpha(1+\alpha)}}}^{P^{\frac{1}{\alpha(1+\alpha)}} T} \frac{d x}{\left|\zeta-\left(x^{\alpha}, 0,0\right)\right|}-\int_{P^{\frac{1}{\alpha(1+\alpha)}} S}^{P^{\frac{1}{\alpha(1+\alpha)}} T} \frac{d x}{|\zeta|}\right| \\
\leq & \int_{P^{\frac{1}{\alpha(1+\alpha)}} S}^{P^{\frac{1}{\alpha(1+\alpha)}} T} \frac{2 x^{\alpha}|\zeta|+x^{2 \alpha}}{\left|\zeta-\left(x^{\alpha}, 0,0\right)\right||\zeta|\left(\left|\zeta-\left(x^{\alpha}, 0,0\right)\right|+|\zeta|\right)} d x \\
\leq & \int_{P^{\frac{1}{\alpha(1+\alpha)}} S}^{P^{\frac{1}{\alpha(1+\alpha)}} T} \frac{2 x^{\alpha}}{D^{2}} d x+\int_{P^{\frac{1}{\alpha(1+\alpha)}} S}^{P^{\frac{1}{\alpha(1+\alpha)}} T} \frac{x^{2 \alpha}}{D^{3}} d x \\
\leq & C_{\alpha} \frac{P^{\frac{1}{\alpha}}\left(T^{\alpha+1}-S^{\alpha+1}\right)+P^{\frac{2 \alpha+1}{\alpha(1+\alpha)}}\left(T^{2 \alpha+1}-S^{2 \alpha+1}\right)}{D^{3}} \\
= & C_{\alpha} \theta^{1+\frac{1}{\alpha}} T^{\alpha+1}(T-S)^{1+\frac{1}{\alpha}} \frac{1-(S / T)^{\alpha+1}+\theta(T-S) T^{\alpha}\left(1-(S / T)^{2 \alpha+1}\right)}{D^{3}}
\end{aligned}
$$

By the similar argument to Proposition 8.3 , we can replace $1-(S / T)^{\alpha+1}$ or $1-(S / T)^{2 \alpha+1}$ by $1-S / T$, hence we obtain the assertion.

\section{Proposition 8.8.}

$$
A_{S^{\prime}, P}^{T^{\prime}} \geq \frac{1}{\theta\left(1+\theta T^{\alpha}(T-S)\right)}, \quad \Phi_{S^{\prime}, P}^{T^{\prime}}\left(\zeta_{\mathbb{R}}, \zeta_{\mathbb{C}}\right) \leq \frac{1}{\theta\left|\zeta_{\mathbb{C}}\right|}
$$

holds for any $\zeta=\left(\zeta_{\mathbb{R}}, \zeta_{\mathbb{C}}\right) \in \mathbb{R}^{3}=\mathbb{R} \oplus \mathbb{C}$.

Proof. One can see

$$
\begin{aligned}
A_{S^{\prime}, P}^{T^{\prime}}=P^{-\frac{1}{1+\alpha}} \int_{S}^{T} \frac{d x}{1+P^{\frac{1}{1+\alpha}} x^{\alpha}} & \geq P^{-\frac{1}{1+\alpha}} \int_{S}^{T} \frac{d x}{1+P^{\frac{1}{1+\alpha}} T^{\alpha}} \\
& \geq \frac{T-S}{P^{\frac{1}{1+\alpha}}\left(1+P^{\frac{1}{1+\alpha}} T^{\alpha}\right)} \\
& =\frac{1}{\theta\left(1+\theta T^{\alpha}(T-S)\right)} .
\end{aligned}
$$


We can also obtain

$$
\Phi_{S^{\prime}, P}^{T^{\prime}}(\zeta) \leq \frac{T-S}{P^{\frac{1}{1+\alpha}}\left|\zeta_{\mathbb{C}}\right|}=\frac{1}{\theta\left|\zeta_{\mathbb{C}}\right|}
$$

Combining Propositions 8.7 and 8.8, the next proposition is obtained.

Proposition 8.9. Let $\Phi=\Phi_{S^{\prime}, P}^{T^{\prime}}$ and $\Phi_{\infty}(\zeta)=\frac{1}{\theta|\zeta|}$. Then $\Phi, \Phi_{\infty}$ satisfy (A3-6) for $R \geq 1$ and

$$
\begin{gathered}
m=3, \quad \varepsilon=\left(1+\theta T^{\alpha}(T-S)\right) T^{\alpha}(T-S), \\
C_{0}=\frac{1}{\theta\left(1+\theta T^{\alpha}(T-S)\right)}, \quad C_{1}=\frac{2}{\theta}, \quad \kappa=0,
\end{gathered}
$$

for any $0 \leq S<T$.

By Propositions 7.14 and 8.9 for $\theta=1$, we have the next result.

Theorem 8.10. Let $\left\{S_{i}\right\}_{i}$ and $\left\{T_{i}\right\}_{i}$ be a sequence such that $0 \leq S_{i}<T_{i}$ and $\lim _{i \rightarrow \infty} T_{i}^{\alpha}\left|T_{i}-S_{i}\right|=0$, then $\left\{\left(\mathbb{R}^{3}, d_{S_{i}}^{T_{i}}, 0\right)\right\}_{i}$ converges to $\left(\mathbb{R}^{3}, \frac{1}{|\zeta|} h_{0}, 0\right)$ in the pointed Gromov-Hausdorff topology.

\subsection{Convergence (3)}

Here, we fix $a>0$ and $n$ and suppose that $T_{a, n}=a^{\frac{1}{1+\alpha}} K_{2 n+1}$ is sufficiently small and $S_{a, n+1}=a^{\frac{1}{1+\alpha}} K_{2 n+2}$ is sufficiently large. Fix $P$ and $\theta$ such that

$$
P^{\frac{1}{1+\alpha}}=\theta\left(T_{a, n}-S_{a, n}\right)=\sqrt{S_{a, n+1}^{-\alpha+1}-T_{a, n+1}^{-\alpha+1}} .
$$

Put $S_{l}^{\prime}=P^{\frac{-1}{1+\alpha}} S_{a, l}$ and $T_{l}^{\prime}=P^{\frac{-1}{1+\alpha}} T_{a, l}$.

Proposition 8.11. Let $R \geq 1$ and $D \leq 1$, and $P$ be as above. Assume $P^{\frac{1}{\alpha(1+\alpha)}} S_{a, n+2} \geq(2 R)^{\frac{1}{\alpha}}$. Then there exists a constant $C_{\alpha}>0$ depending only on $\alpha$ such that

$$
\left|\Phi_{a}(\zeta)-\Phi_{S_{n}^{\prime}, P}^{T_{n}^{\prime}}(\zeta)-\Phi_{S_{n+1}^{\prime}, P}^{T_{n+1}^{\prime}}(\zeta)\right| \leq \frac{C_{\alpha} \varepsilon_{a, n}}{D}
$$

for any $\zeta \in K(R, D)$, where $\varepsilon_{a, n}$ is the constant defined by

$$
\varepsilon_{a, n}=\frac{1+K_{2 n-1}}{\theta\left(K_{2 n+1}-K_{2 n}\right)}+\frac{K_{2 n+4}^{-\alpha+1}}{K_{2 n+2}^{-\alpha+1}-K_{2 n+3}^{-\alpha+1}} .
$$


Proof. By Propositions 6.1 and (7) (8)), we have

$$
\left|\Phi_{a}-\Phi_{S_{n}^{\prime}, P}^{T_{n}^{\prime}}-\Phi_{S_{n+1}^{\prime}, P}^{T_{n+1}^{\prime}}\right| \leq \frac{2\left(\frac{a}{P}\right)^{\frac{1}{1+\alpha}}+P^{\frac{-1}{1+\alpha}} T_{a, n-1}}{D}+\frac{2 S_{a, n+2}^{-\alpha+1}}{P^{\frac{2}{1+\alpha}}(\alpha-1)},
$$

if $P^{\frac{1}{\alpha(1+\alpha)}} S_{a, n+2} \geq(2 R)^{\frac{1}{\alpha}}$. Since we have

$$
\begin{aligned}
\left(\frac{a}{P}\right)^{\frac{1}{1+\alpha}} & =\frac{1}{\theta\left(K_{2 n+1}-K_{2 n}\right)}, \\
P^{\frac{-1}{1+\alpha}} T_{a, n-1} & =\frac{K_{2 n-1}}{\theta\left(K_{2 n+1}-K_{2 n}\right)}, \\
\frac{S_{a, n+2}^{-\alpha+1}}{P^{\frac{2}{1+\alpha}}} & =\frac{K_{2 n+4}^{-\alpha+1}}{K_{2 n+2}^{-\alpha+1}-K_{2 n+3}^{-\alpha+1}},
\end{aligned}
$$

then we have the assertion.

Here, the assumption $P^{\frac{1}{\alpha(1+\alpha)}} S_{a, n+2} \geq(2 R)^{\frac{1}{\alpha}}$ can be replaced by

$$
\left(\frac{K_{2 n+4}}{K_{2 n+2}}\right)^{\alpha} S_{a, n+1}^{\alpha} \sqrt{S_{a, n+1}^{-\alpha+1}-T_{a, n+1}^{-\alpha+1}} \geq 2 R .
$$

We can apply Propositions 8.3 and 8.7 to $\Phi_{S_{n}^{\prime}, P}^{T_{n}^{\prime}}$ and $\Phi_{S_{n+1}^{\prime}, P}^{T_{n+1}^{\prime}}$. If we put

$$
S=S_{a, n+1}, \quad T=T_{a, n+1}, \quad \theta=1, \quad P^{\frac{1}{1+\alpha}}=\sqrt{S_{a, n+1}^{-\alpha+1}-T_{a, n+1}^{-\alpha+1}},
$$

in Proposition 8.3, then we have

$$
\left|\Phi_{S_{n+1}^{\prime}, P}^{T_{n+1}^{\prime}}-\frac{1}{\alpha-1}\right| \leq \frac{C R}{S_{a, n+1}^{\alpha} \sqrt{S_{a, n+1}^{-\alpha+1}-T_{a, n+1}^{-\alpha+1}}}
$$

for any $\zeta \in K(R, D)$ if $S_{a, n+1}^{\alpha} \sqrt{S_{a, n+1}^{-\alpha+1}-T_{a, n+1}^{-\alpha+1}} \geq 2 R$.

If we put

$$
S=S_{a, n}, \quad T=T_{a, n}, \quad P^{\frac{1}{1+\alpha}}=\theta\left(T_{a, n}-S_{a, n}\right),
$$

in Proposition 8.7, then we have

$$
\begin{aligned}
& \left|\Phi_{S_{n}^{\prime}, P}^{T_{n}^{\prime}}-\frac{1}{\theta|\zeta|}\right| \leq \frac{2}{\theta D}, \\
& \left|\Phi_{S_{n}^{\prime}, P}^{T_{n}^{\prime}}-\frac{1}{\theta|\zeta|}\right| \leq \frac{1+\theta T_{a, n}^{\alpha}\left(T_{a, n}-S_{a, n}\right)}{D^{3}} T_{a, n}^{\alpha}\left(T_{a, n}-S_{a, n}\right) .
\end{aligned}
$$


Now, we put $\Phi=\Phi_{a}, \Phi_{\infty}=\frac{1}{\alpha-1}+\frac{1}{\theta|\zeta|}$. Combining above arguments and Proposition 8.11, we can describe $\varepsilon, C_{1}$ in (A3) explicitly, with $m=3$. Moreover, by Propositions 8.3, 8.7, 8.4 and 8.8, we obtain $C_{0}, C_{1}$ in (A5-6) and $\kappa=1$. Fix a constant $A>0$ and suppose

$$
A^{-1} \leq \theta \leq A, \quad S_{a, n+1}^{\alpha} \sqrt{S_{a, n+1}^{-\alpha+1}-T_{a, n+1}^{-\alpha+1}} \geq 2 R,
$$

and and $P$ is as above. Then we can take these constants in (A3-6) being only depending on $\alpha, A, R$, if $\varepsilon_{a, n}, S_{a, n+1}^{-\alpha}\left(S_{a, n+1}^{-\alpha+1}-T_{a, n+1}^{-\alpha+1}\right)^{\frac{-1}{2}}$ and $T_{a, n}^{\alpha}\left(T_{a, n}-\right.$ $\left.S_{a, n}\right)$ are sufficiently small. Therefore, we obtain the following result.

Theorem 8.12. Let $\left(X, g_{\Lambda}\right)$ be as in Section 6, take a subsequence

$$
\left\{K_{n_{0}}<K_{n_{1}}<K_{n_{2}}<\cdots\right\} \subset\left\{K_{0}<K_{1}<K_{2}<\cdots\right\}
$$

and suppose

$$
\lim _{i \rightarrow \infty}\left\{\frac{K_{2 n_{i}-1}}{K_{2 n_{i}+1}-K_{2 n_{i}}}+\frac{K_{2 n_{i}+4}^{-\alpha+1}}{K_{2 n_{i}+2}^{-\alpha+1}-K_{2 n_{i}+3}^{-\alpha+1}}\right\}=0 .
$$

If a sequence $\left\{a_{i}\right\}_{i} \subset \mathbb{R}^{+}$satisfies

$$
\begin{aligned}
& \lim _{i \rightarrow \infty} \frac{\sqrt{S_{a_{i}, n_{i}+1}^{-\alpha+1}-T_{a_{i}, n_{i}+1}^{-\alpha+1}}}{T_{a_{i}, n_{i}}-S_{a_{i}, n_{i}}}=\theta>0, \\
& \lim _{i \rightarrow \infty} S_{a_{i}, n_{i}+1}^{-\alpha}\left(S_{a_{i}, n_{i}+1}^{-\alpha+1}-T_{a_{i}, n_{i}+1}^{-\alpha+1}\right)^{\frac{-1}{2}}=\lim _{i \rightarrow \infty} T_{a_{i}, n_{i}}^{\alpha}\left(T_{a_{i}, n_{i}}-S_{a_{i}, n_{i}}\right)=0,
\end{aligned}
$$

then $\left\{\left(X, a_{i} g_{\Lambda}, p\right)\right\}_{n} \stackrel{G H}{\longrightarrow}\left(\mathbb{R}^{3},\left(\frac{1}{\alpha-1}+\frac{1}{\theta|\zeta|}\right) h_{0}, 0\right)$.

Next we estimate $\Phi_{a}-\frac{1}{\alpha-1}$ in the same situation, as $\theta \rightarrow \infty$. We have

$$
\begin{aligned}
\left|\Phi_{a}-\Phi_{S_{n+1}^{\prime}, P}^{T_{n+1}^{\prime}}\right| & \leq \frac{2\left(\frac{a}{P}\right)^{\frac{1}{1+\alpha}}+P^{\frac{-1}{1+\alpha}} T_{a, n-1}+P^{\frac{-1}{1+\alpha}}\left(T_{a, n}-S_{a, n}\right)}{D}+\frac{2 S_{a, n+2}^{-\alpha+1}}{P^{\frac{2}{1+\alpha}}(\alpha-1)} \\
& \leq \frac{C_{\alpha}}{D}\left\{\frac{1+K_{2 n-1}}{\theta\left(K_{2 n+1}-K_{2 n}\right)}+\frac{1}{\theta}+\frac{K_{2 n+4}^{-\alpha+1}}{K_{2 n+2}^{-\alpha+1}-K_{2 n+3}^{-\alpha+1}}\right\} .
\end{aligned}
$$


Applying Propositions 8.3 and 8.4 with $\theta=1$ and (5) , we have

$$
\begin{aligned}
\left|\Phi_{a}-\frac{1}{\alpha-1}\right| \leq & \frac{C_{\alpha}}{D}\left\{\frac{1+K_{2 n-1}}{\theta\left(K_{2 n+1}-K_{2 n}\right)}+\frac{1}{\theta}+\frac{K_{2 n+4}^{-\alpha+1}}{K_{2 n+2}^{-\alpha+1}-K_{2 n+3}^{-\alpha+1}}\right. \\
& \left.+\frac{R}{S_{a, n+1}^{\alpha} \sqrt{S_{a, n+1}^{-\alpha+1}-T_{a, n+1}^{-\alpha+1}}}\right\} \\
\Phi_{a} \geq & \left(A_{S_{n+1}^{\prime}, P}^{T_{n+1}^{\prime}}-\frac{2}{\theta\left(K_{2 n+1}-K_{2 n}\right)}\right) \min \left\{\frac{1}{|\zeta|}, 1\right\} \\
\geq & \left(\frac{1}{2(\alpha-1)}-\frac{2}{\theta\left(K_{2 n+1}-K_{2 n}\right)}\right) \min \left\{\frac{1}{|\zeta|}, 1\right\}
\end{aligned}
$$

if $D \leq 1, R \geq 1$ and $|\zeta| \leq R$. Therefore, we can take $C_{0}, C_{1}, \kappa, m$ in (A3-6) depending only on $\alpha, R$ if $\varepsilon \rightarrow 0$, where $\Phi=\Phi_{a}, \Phi_{\infty}=\frac{1}{\alpha-1}$, hence we have the following theorem.

Theorem 8.13. Let $\left(X, g_{\Lambda}\right)$ be as in Section 6 and suppose $\left\{K_{n_{i}}\right\}_{i}$ satisfies (16). If a sequence $\left\{a_{i}\right\}_{i} \subset \mathbb{R}^{+}$satisfies

$$
\begin{aligned}
& \lim _{i \rightarrow \infty} \frac{\sqrt{S_{a_{i}, n_{i}+1}^{-\alpha+1}-T_{a_{i}, n_{i}+1}^{-\alpha+1}}}{T_{a_{i}, n_{i}}-S_{a_{i}, n_{i}}}=\infty, \\
& \lim _{i \rightarrow \infty} S_{a_{i}, n_{i}+1}^{-\alpha}\left(S_{a_{i}, n_{i}+1}^{-\alpha+1}-T_{a_{i}, n_{i}+1}^{-\alpha+1}\right)^{\frac{-1}{2}}=0,
\end{aligned}
$$

then $\left\{\left(X, a_{i} g_{\Lambda}, p\right)\right\}_{n} \stackrel{G H}{\longrightarrow}\left(\mathbb{R}^{3}, h_{0}, 0\right)$.

By the similar argument, we have the following.

Theorem 8.14. Let $\left(X, g_{\Lambda}\right)$ be as in Section 6 and suppose $\left\{K_{n_{i}}\right\}_{i}$ satisfies (16). If a sequence $\left\{a_{i}\right\}_{i} \subset \mathbb{R}^{+}$satisfies

$$
\begin{aligned}
& \lim _{i \rightarrow \infty} \frac{\sqrt{S_{a_{i}, n_{i}+1}^{-\alpha+1}-T_{a_{i}, n_{i}+1}^{-\alpha+1}}}{T_{a_{i}, n_{i}}-S_{a_{i}, n_{i}}}=0, \\
& \lim _{i \rightarrow \infty} S_{a_{i}, n_{i}+1}^{-\alpha}\left(S_{a_{i}, n_{i}+1}^{-\alpha+1}-T_{a_{i}, n_{i}+1}^{-\alpha+1}\right)^{\frac{-1}{2}}=\lim _{i \rightarrow \infty} T_{a_{i}, n_{i}}^{\alpha}\left(T_{a_{i}, n_{i}}-S_{a_{i}, n_{i}}\right)=0,
\end{aligned}
$$

then $\left\{\left(X, a_{i} g_{\Lambda}, p\right)\right\}_{n} \stackrel{G H}{\longrightarrow}\left(\mathbb{R}^{3}, \frac{1}{|\zeta|} h_{0}, 0\right)$.

Proof. Put

$$
\begin{aligned}
P^{\frac{1}{1+\alpha}} & :=\left(T_{a, n}-S_{a, n}\right)=\theta \sqrt{S_{a, n+1}^{-\alpha+1}-T_{a, n+1}^{-\alpha+1}}, \\
S_{l}^{\prime} & =P^{\frac{-1}{1+\alpha}} S_{a, l}, \quad T_{l}^{\prime}=P^{\frac{-1}{1+\alpha}} T_{a, l} .
\end{aligned}
$$


The similar argument to (7) gives

$$
\Phi_{S_{n+1}^{\prime}, P}^{T_{n+1}^{\prime}}(\zeta) \leq \frac{2\left(\left(S_{n+1}^{\prime}\right)^{-\alpha+1}-\left(T_{n+1}^{\prime}\right)^{-\alpha+1}\right)}{P(\alpha-1)}
$$

if $P\left(S_{n+1}^{\prime}\right)^{\alpha} \geq 2 R$, which is equivalent to $\theta S_{a, n+1}^{\alpha} \sqrt{S_{a, n+1}^{-\alpha+1}-T_{a, n+1}^{-\alpha+1}} \geq 2 R$, then the similar argument to Proposition 8.11 gives

$$
\left|\Phi_{a}(\zeta)-\Phi_{S_{n}^{\prime}, P}^{T_{n}^{\prime}}(\zeta)\right| \leq \frac{C_{\alpha} \varepsilon_{a, n}}{D}+\frac{2}{(\alpha-1) \theta}
$$

for any $\zeta \in K(R, D)$, where $\varepsilon_{a, n}$ is the constant defined by

$$
\varepsilon_{a, n}=\frac{1+K_{2 n-1}}{K_{2 n+1}-K_{2 n}}+\frac{K_{2 n+4}^{-\alpha+1}}{\theta^{2}\left(K_{2 n+2}^{-\alpha+1}-K_{2 n+3}^{-\alpha+1}\right)} .
$$

Moreover, Proposition 8.7 with $\theta=1$ gives

$$
\begin{aligned}
\left|\Phi_{S_{n}^{\prime}, P}^{T_{n}^{\prime}}(\zeta)-\frac{1}{|\zeta|}\right| & \leq \frac{2}{D} \\
\left|\Phi_{S_{n}^{\prime}, P}^{T_{n}^{\prime}}(\zeta)-\frac{1}{|\zeta|}\right| & \leq \frac{1+T_{a, n}^{\alpha}\left(T_{a, n}-S_{a, n}\right)}{D^{3}} T_{a, n}^{\alpha}\left(T_{a, n}-S_{a, n}\right) .
\end{aligned}
$$

Then we can see $\left|\Phi_{a}-\frac{1}{|\zeta|}\right| \leq \frac{\varepsilon}{D^{3}}$ for some $\varepsilon>0$ if $D \leq 1$ and $\zeta \in K(R, D)$. Here, $\varepsilon$ goes to 0 as $\varepsilon_{a, n} \rightarrow 0, \theta \rightarrow \infty, S_{a, n+1}^{\alpha} \sqrt{S_{a, n+1}^{-\alpha+1}-T_{a, n+1}^{-\alpha+1}} \rightarrow \infty$ and $T_{a, n}^{\alpha}\left(T_{a, n}-S_{a, n}\right) \rightarrow 0$. Since one can take $C_{0}, C_{1}, m, \kappa$ in (A3-6) depending only on $\alpha$ if $\varepsilon$ is sufficiently small, by Proposition 8.8 with $\theta=1$ and (5).

\subsection{Example (1)}

Let $\Lambda$ be as in Section 6. Moreover we take and increasing sequence $\left\{K_{n}\right\}_{n}$ such that

$$
\lim _{n \rightarrow \infty} \frac{K_{n}}{K_{n-1}}=\infty
$$

In this situation, we observe which pointed metric spaces can be contained in $\mathcal{T}\left(X, g_{\Lambda}\right)$ and prove Theorem 1.2 ,

Take $S>0$ and put $a_{i}:=K_{2 i}^{-1-\alpha} S^{1+\alpha}$. Then we have $a_{i}^{\frac{1}{1+\alpha}} K_{2 i}=S$ and $\lim _{i} a_{i}^{\frac{1}{1+\alpha}} K_{2 i+1}=\infty$. Hence Theorem 8.2 implies that $\left(X, a_{i} g_{\Lambda}, p\right) \stackrel{G H}{\longrightarrow}$ $\left(\mathbb{R}^{3}, d_{S}^{\infty}, 0\right)$. Similarly, if we take $a_{i}:=K_{2 i+1}^{-1-\alpha} T^{1+\alpha}$ for $T>0$ then we obtain $\left(\mathbb{R}^{3}, d_{0}^{T}, 0\right)$ as the pointed Gromov-Hausdorff limit. 
Next we fix $\theta>0$ and put $a_{i}=\theta^{-1} K_{2 i+1}^{-2} K_{2 i+2}^{-\alpha+1}$. Then one can check that the assumptions of Theorem 8.12 is satisfied, hence one obtain $\left(\mathbb{R}^{3},\left(\frac{1}{\alpha-1}+\right.\right.$ $\left.\left.\frac{1}{\theta|\zeta|}\right) h_{0}, 0\right)$ as the pointed Gromov-Hausdorff limit. By taking the limit $\theta \rightarrow 0$ or $\theta \rightarrow \infty$, we obtain $\left(\mathbb{R}^{3}, h_{0}, 0\right)$ and $\left(\mathbb{R}^{3}, \frac{1}{|\zeta|} h_{0}, 0\right)$ as the pointed GromovHausdorff limit.

In fact, we obtain the next result.

Theorem 8.15. Let $\Lambda,\left\{K_{n}\right\}_{n}$ satisfy $\lim _{n \rightarrow \infty} \frac{K_{n}}{K_{n-1}}=\infty$. Then $\mathcal{T}\left(X, g_{\Lambda}\right)$ is equal to the closure of

$$
\left\{\left(\mathbb{R}^{3}, s d_{1}^{\infty}, 0\right) ; s>0\right\} \cup\left\{\left(\mathbb{R}^{3}, s d_{0}^{1}, 0\right) ; s>0\right\} \cup\left\{\left(\mathbb{R}^{3}, s\left(1+\frac{1}{|\zeta|}\right) h_{0}, 0\right) ; s>0\right\}
$$

with respect to the Gromov-Hausdorff topology. Moreover we have

$$
\begin{aligned}
& \lim _{s \rightarrow \infty}\left(\mathbb{R}^{3}, s d_{1}^{\infty}, 0\right)=\lim _{s \rightarrow 0}\left(\mathbb{R}^{3}, s\left(1+\frac{1}{|\zeta|}\right) h_{0}, 0\right)=\left(\mathbb{R}^{3}, h_{0}, 0\right), \\
& \lim _{s \rightarrow 0}\left(\mathbb{R}^{3}, s d_{0}^{1}, 0\right)=\lim _{s \rightarrow \infty}\left(\mathbb{R}^{3}, s\left(1+\frac{1}{|\zeta|}\right), 0\right)=\left(\mathbb{R}^{3}, \frac{1}{|\zeta|} h_{0}, 0\right), \\
& \lim _{s \rightarrow 0}\left(\mathbb{R}^{3}, s d_{1}^{\infty}, 0\right)=\lim _{s \rightarrow \infty}\left(\mathbb{R}^{3}, s d_{0}^{1}, 0\right)=\left(\mathbb{R}^{3}, d_{0}^{\infty}, 0\right) .
\end{aligned}
$$

Proof. We have already shown that the pointed metric spaces in the above list are contained in $\mathcal{T}\left(X, g_{\Lambda}\right)$. Accordingly, what we have to show is that any other pointed metric spaces may not arise as the tangent cone at infinity of $\left(X, g_{\Lambda}\right)$.

Suppose that a sequence $\left\{a_{i}\right\}_{i} \subset \mathbb{R}^{+}$is given such that $\left(X, a_{i} g_{\Lambda}, p\right) \stackrel{G H}{\longrightarrow}$ $(Y, d, q)$ as $i \rightarrow \infty$. It suffices to show that $(Y, d, q)$ is one of the metric spaces in the list.

First of all, we may assume that for any large $M>0$ there exists $i(M)$ such that

$$
\left\{a_{i}^{\frac{1}{1+\alpha}} K_{n} \in \mathbb{R}^{+} ; n \in \mathbb{N}\right\} \cap\left[M^{-1}, M\right]
$$

is empty for any $i \geq i(M)$. If not, there is $M>0$ and a map $i \mapsto n_{i}$ such that $M^{-1} \leq a_{i}^{\frac{1}{1+\alpha}} K_{n_{i}} \leq M$ holds for infinitely many $i$. Then by taking subsequence $\left\{a_{i_{j}}\right\} \subset\left\{a_{i}\right\}_{i}$, we may suppose $M^{-1} \leq a_{i_{j}}^{\frac{1}{1+\alpha}} K_{2 n_{i_{j}}} \leq M$ holds for any $j$ or $M^{-1} \leq a_{i_{j}}^{\frac{1}{1+\alpha}} K_{2 n_{i_{j}}+1} \leq M$ holds for any $j$. If the former case holds, then by replacing by subsequence we may suppose

$$
\begin{aligned}
\lim _{i \rightarrow \infty} a_{i}^{\frac{1}{1+\alpha}} K_{2 n_{i}} & =S \in\left[M^{-1}, M\right], \\
\lim _{i \rightarrow \infty} a_{i}^{\frac{1}{1+\alpha}} K_{2 n_{i}+1} & =S \lim _{i \rightarrow \infty} \frac{K_{2 n_{i}+1}}{K_{2 n_{i}}}=\infty,
\end{aligned}
$$


and we can apply Theorem 8.2 hence obtain $(Y, d, q)=\left(\mathbb{R}^{3}, d_{S}^{\infty}, 0\right)$. If the latter case holds, then we have $(Y, d, q)=\left(\mathbb{R}^{3}, d_{0}^{T}, 0\right)$ for some $T>0$.

Now, we may suppose that there exists $l_{i} \in \mathbb{N}$ for each $i$ such that $\lim _{i \rightarrow \infty} a_{i}^{\frac{1}{1+\alpha}} K_{l_{i}}=0$ and $\lim _{i \rightarrow \infty} a_{i}^{\frac{1}{1+\alpha}} K_{l_{i}+1}=\infty$ hold. If $\left\{i \in \mathbb{N} ; l_{i}\right.$ is even. $\}$ is an infinite set, then we can apply Theorem 8.2 again and obtain $(Y, d, q)=$ $\left(\mathbb{R}^{3}, d_{0}^{\infty}, 0\right)$. Therefore, replacing by subsequence, we may suppose

$$
\lim _{i \rightarrow \infty} a_{i}^{\frac{1}{1+\alpha}} K_{2 n_{i}+1}=0, \quad \lim _{i \rightarrow \infty} a_{i}^{\frac{1}{1+\alpha}} K_{2 n_{i}+2}=\infty .
$$

Now, we have

$$
\sqrt{S_{a, n+1}^{-\alpha+1}-T_{a, n+1}^{-\alpha+1}} \geq \frac{S_{a, n+1}^{\frac{-\alpha+1}{2}}}{2}, \quad T_{a, n}-S_{a, n} \geq \frac{T_{a, n}}{2}
$$

holds for sufficiently large $n$. Hence if

$$
0<\liminf _{i \rightarrow \infty} \frac{S_{a_{i}, n_{i}+1}^{\frac{1-\alpha}{2}}}{T_{a_{i}, n_{i}}} \leq \limsup _{i \rightarrow \infty} \frac{S_{a_{i}, n_{i}+1}^{\frac{1-\alpha}{2}}}{T_{a_{i}, n_{i}}}<\infty
$$

holds, then Theorem 8.12 can be applied to this situation by taking a subsequence, then we obtain $(Y, d, q)=\left(\mathbb{R}^{3},\left(1+\frac{\theta}{|\zeta|}\right) h_{0}, 0\right)$ for some $\theta>0$. Hence the remaining cases are

$$
\lim _{i \rightarrow \infty} \frac{S_{a_{i}, n_{i}+1}^{\frac{1-\alpha}{2}}}{T_{a_{i}, n_{i}}}=0 \quad \text { or } \quad \lim _{i \rightarrow \infty} \frac{S_{a_{i}, n_{i}+1}^{\frac{1-\alpha}{2}}}{T_{a_{i}, n_{i}}}=\infty .
$$

In both of the cases, we can apply Theorems 8.13 or 8.14 , then obtain $(Y, d, q)=\left(\mathbb{R}^{3}, h_{0}, 0\right)$ or $\left(\mathbb{R}^{3}, \frac{1}{|\zeta|} h_{0}, 0\right)$.

One can also see that there are no nontrivial isometries between two pointed metric spaces appearing in the list of Theorem 8.15. Here, the isometry of pointed metric spaces means the bijective morphism preserving the metrics and the base points.

Obviously, there is no isometry between $\left(\mathbb{R}^{3}, h_{0}, 0\right)$ and $\left(\mathbb{R}^{3}, \frac{1}{|\zeta|} h_{0}, 0\right)$. In the next section, we show that $\left(\mathbb{R}^{3}, d_{0}^{\infty}, 0\right)$ is isometric to neither $\left(\mathbb{R}^{3}, h_{0}, 0\right)$ nor $\left(\mathbb{R}^{3}, \frac{1}{|\zeta|} h_{0}, 0\right)$.

Then, Table 1 implies that the nontrivial isometries may exist between

$$
\begin{gathered}
\left(\mathbb{R}^{3}, d_{S}^{\infty}, 0\right) \text { and }\left(\mathbb{R}^{3}, d_{S^{\prime}}^{\infty}, 0\right) \text { for } S \neq S^{\prime}, \\
\left(\mathbb{R}^{3}, d_{0}^{T}, 0\right) \text { and }\left(\mathbb{R}^{3}, d_{0}^{T^{\prime}}, 0\right) \text { for } T \neq T^{\prime}, \\
\left(\mathbb{R}^{3},\left(1+\frac{\theta}{|\zeta|}\right) h_{0}, 0\right) \text { and }\left(\mathbb{R}^{3},\left(1+\frac{\theta^{\prime}}{|\zeta|}\right) h_{0}, 0\right) \text { for } \theta \neq \theta^{\prime}
\end{gathered}
$$


Table 1: Tangent cones $(0<S, T, \theta<\infty)$

\begin{tabular}{|c||c|c|}
\hline metric & tangent cone at 0 & tangent cone at $\infty$ \\
\hline \hline$d_{S}^{T}(S<T)$ & $h_{0}$ & $\frac{1}{|\zeta|} h_{0}$ \\
\hline$d_{S}^{\infty}$ & $h_{0}$ & $d_{0}^{\infty}$ \\
\hline$d_{0}^{T}$ & $d_{0}^{\infty}$ & $\frac{1}{|\zeta|} h_{0}$ \\
\hline$d_{0}^{\infty}$ & $d_{0}^{\infty}$ & $d_{0}^{\infty}$ \\
\hline$h_{0}$ & $h_{0}$ & $h_{0}$ \\
\hline$\frac{1}{|\zeta|} h_{0}$ & $\frac{1}{|\zeta|} h_{0}$ & $\frac{1}{|\zeta|} h_{0}$ \\
\hline$\left(1+\frac{\theta}{|\zeta|}\right) h_{0}$ & $\frac{1}{|\zeta|} h_{0}$ & $h_{0}$ \\
\hline
\end{tabular}

Suppose $\left(\mathbb{R}^{3}, d_{S}^{\infty}, 0\right)$ is isometric to $\left(\mathbb{R}^{3}, d_{S^{\prime}}^{\infty}, 0\right)$ for some $S \neq S^{\prime}$. Then the topological space

$$
\left\{\left(\mathbb{R}^{3}, d_{S}^{\infty}, 0\right) ; S \in \mathbb{R}^{+}\right\}
$$

with respect to pointed Gromov-Hausdorff topology is homeomorphic to $S^{1}$ or 1-point, hence it is compact. Then its closure is itself, therefore $\left(\mathbb{R}^{3}, h_{0}, 0\right)$ is isometric to some $\left(\mathbb{R}^{3}, d_{S}^{\infty}, 0\right)$, which is the contradiction by Table 1 . Similarly, we can show that there are no isometries between $\left(\mathbb{R}^{3}, d_{0}^{T}, 0\right)$ and $\left(\mathbb{R}^{3}, d_{0}^{T^{\prime}}, 0\right)$, and between $\left(\mathbb{R}^{3},\left(1+\frac{\theta}{|\zeta|}\right) h_{0}, 0\right)$ and $\left(\mathbb{R}^{3},\left(1+\frac{\theta^{\prime}}{|\zeta|}\right) h_{0}, 0\right)$.

\subsection{Example (2)}

Next we suppose that $\left\{K_{n}\right\}_{n}$ satisfies

$$
\lim _{n \rightarrow \infty} \frac{K_{2 n}}{K_{2 n-1}}=\infty, \quad \frac{K_{2 n+1}}{K_{2 n}}=\beta>1 .
$$

Take $S>0$ and put $a_{n}:=K_{2 n}^{-1-\alpha} S^{1+\alpha}$. Then we have $a_{n}^{\frac{1}{1+\alpha}} K_{2 n}=S$ and $a_{n}^{\frac{1}{1+\alpha}} K_{2 n+1}=\beta S$. Hence Theorem 8.2 implies that $\left(X, a_{n} g_{\Lambda}, p\right) \stackrel{G H}{\longrightarrow}$ $\left(\mathbb{R}^{3}, d_{S}^{\beta S}\right)$. By arguing similarly to the proof of Theorem 8.15 we obtain the followings.

Theorem 8.16. Let $\Lambda,\left\{K_{n}\right\}_{n}$ satisfy

$$
\lim _{n \rightarrow \infty} \frac{K_{2 n}}{K_{2 n-1}}=\infty, \quad \lim _{n \rightarrow \infty} \frac{K_{2 n+1}}{K_{2 n}}=\beta>1 .
$$

Then $\mathcal{T}\left(X, g_{\Lambda}\right)$ is equal to the closure of

$$
\left\{\left(\mathbb{R}^{3}, s d_{1}^{\beta}, 0\right) ; s>0\right\} \cup\left\{\left(\mathbb{R}^{3}, s\left(1+\frac{1}{|\zeta|}\right) h_{0}, 0\right) ; s>0\right\}
$$


with respect to the Gromov-Hausdorff topology. Moreover we have

$$
\begin{aligned}
& \lim _{s \rightarrow \infty}\left(\mathbb{R}^{3}, s d_{1}^{\beta}, 0\right)=\lim _{s \rightarrow 0}\left(\mathbb{R}^{3}, s\left(1+\frac{1}{|\zeta|}\right) h_{0}, 0\right)=\left(\mathbb{R}^{3}, h_{0}, 0\right), \\
& \lim _{s \rightarrow 0}\left(\mathbb{R}^{3}, s d_{1}^{\beta}, 0\right)=\lim _{s \rightarrow \infty}\left(\mathbb{R}^{3}, s\left(1+\frac{1}{|\zeta|}\right), 0\right)=\left(\mathbb{R}^{3}, \frac{1}{|\zeta|} h_{0}, 0\right) .
\end{aligned}
$$

By the similar argument to Section 8.4 , we can see that $\left(\mathbb{R}^{3}, d_{S}^{\beta S}, 0\right)$ is isometric to neither $\left(\mathbb{R}^{3}, h_{0}, 0\right),\left(\mathbb{R}^{3}, \frac{1}{|\zeta|} h_{0}, 0\right)$ nor $\left(\mathbb{R}^{3}, d_{S^{\prime}}^{\beta S^{\prime}}, 0\right)$ for $S^{\prime} \neq S$.

\subsection{Example (3)}

For $I \subset \mathbb{R}^{+}$, denote by $d_{I}$ the metric on $\mathbb{R}^{3}$ induced by

$$
\int_{x \in I} \frac{d x}{\left|\zeta-\left(x^{\alpha}, 0,0\right)\right|} \cdot h_{0} .
$$

Denote by $\mathcal{B}_{+}\left(\mathbb{R}^{+}\right)$the set consisting of all Borel subsets of $\mathbb{R}^{+}$of nonzero Lebesgue measure. In this subsection we show the next theorem.

Theorem 8.17. There is a sequence $\left\{K_{n}\right\}_{n}$ such that $\mathcal{T}\left(X, g_{\Lambda}\right)$ contains

$$
\left\{\left(\mathbb{R}^{3}, d_{I}, 0\right) ; I \in \mathcal{B}_{+}\left(\mathbb{R}^{+}\right)\right\} / \text {isometry } .
$$

Proof. Put

$$
\begin{aligned}
& \mathcal{O}_{0}:=\left\{I \subset \mathbb{R}^{+} ; I \text { is nonempty and open }\right\} \text {, } \\
& \mathcal{O}_{1}:=\left\{\begin{array}{ll}
\bigcup_{i=1}^{k}\left(S_{l}, T_{l}\right) \subset \mathbb{R}^{+} ; \begin{array}{l}
S_{l}, T_{l} \in \mathbb{Q}, 1 \leq k<\infty, \\
0<S_{l}<T_{l}<S_{l+1}<\infty
\end{array}
\end{array}\right\},
\end{aligned}
$$

then one can see $\mathcal{O}_{1} \subset \mathcal{O}_{0} \subset \mathcal{B}_{+}\left(\mathbb{R}^{+}\right)$. Since $\mathcal{O}_{1}$ is countable, we can label the open sets in $\mathcal{O}_{1}$ such as

$$
\mathcal{O}_{1}=\left\{I_{1}, I_{2}, I_{3}, \ldots\right\}, \quad I_{m}=\bigcup_{l=1}^{k_{m}}\left(S_{m, l}, T_{m, l}\right) .
$$

Now, we fix a bijection $F: \mathbb{N} \rightarrow \mathbb{N} \times \mathbb{N}$ and write $F(q)=(i(q), m(q))$. Define $L_{q}>0$ inductively by

$$
L_{q+1}:=2^{i(q)+i(q+1)} L_{q} \cdot \frac{T_{m(q), k_{m(q)}}}{S_{m(q), 1}}, \quad L_{0}:=1 .
$$


Then we can define $0<K_{0}<K_{1}<\cdots$ such that

$$
\left\{K_{0}<K_{1}<\cdots\right\}=\left\{L_{q} \frac{S_{m(q), l}}{S_{m(q), 1}}, L_{q} \frac{T_{m(q), l}}{S_{m(q), 1}} ; 1 \leq l \leq k_{m(q)}, q=0,1, \ldots\right\} .
$$

First of all we show $\left(\mathbb{R}^{3}, d_{I_{m}}, 0\right) \in \mathcal{T}\left(X, g_{\Lambda}\right)$ for every $I_{m} \in \mathcal{O}_{1}$. Fix $m$. For any $i \in \mathbb{N}$, we can take a unique $q$ such that $i(q)=i$ and $m(q)=m$. Put $a_{i}^{\frac{1}{1+\alpha}}:=L_{q}^{-1} S_{m, 1}$, then we have

$$
a_{i}^{\frac{1}{1+\alpha}} L_{q} \frac{S_{m, l}}{S_{m, 1}}=S_{m, l}, \quad a_{i}^{\frac{1}{1+\alpha}} L_{q} \frac{T_{m, l}}{S_{m, 1}}=T_{m, l}
$$

Note that $L_{q+1} \geq 2^{i(q)+i(q+1)} L_{q}$ implies $L_{q} \rightarrow \infty$ as $i \rightarrow \infty$, hence $a_{i} \rightarrow 0$ as $i \rightarrow \infty$. Here, we put $\Phi=\Phi_{a_{i}}$ and $\Phi_{\infty}=\sum_{l=1}^{k_{m}} \Phi_{S_{m, l}}^{T_{m, l}}$. By applying Proposition 6.1 and (4)-(8) with $P=1$, the constants appearing in (A3-6) are given by

$$
\begin{aligned}
& \varepsilon=2 a_{i}^{\frac{1}{1+\alpha}}+2^{-i} S_{m, 1}+\frac{2^{1-(\alpha-1) i} T_{m, k_{m}}^{-\alpha+1}}{\alpha-1}, \quad C_{0}=\frac{1}{2} \sum_{l=1}^{k_{m}} A_{S_{m, l}}^{T_{m, l}}, \\
& C_{1}=\frac{\alpha 2^{\frac{1}{\alpha}}}{\alpha-1}, \quad m=1, \quad \kappa=\frac{1}{\alpha},
\end{aligned}
$$

if we suppose $\varepsilon$ is sufficiently small. One can see $\varepsilon \rightarrow 0$ as $i \rightarrow \infty$, then we obtain $\left\{\left(X, a_{i} g_{\Lambda}, p\right)\right\}_{i} \stackrel{\mathrm{GH}}{\longrightarrow}\left(\mathbb{R}^{3}, d_{I_{m}}, 0\right)$.

Next we show that $\left(\mathbb{R}^{3}, d_{I}, 0\right) \in \mathcal{T}\left(X, g_{\Lambda}\right)$ for any $I \in \mathcal{O}_{0}$. To show it, we apply Vitali's Covering Theorem. Fix $I \in \mathcal{O}_{0}$ and put $\mathcal{I}:=\{(a, b) \in$ $\left.\mathcal{O}_{0} ;[a, b] \subset I\right\}$. Then $\mathcal{I}$ is a Vitali cover of $I$, hence there exists $\left\{J_{n}\right\}_{n \in \mathbb{N}} \subset \mathcal{I}$ such that

$$
J_{n} \neq J_{n^{\prime}}\left(\text { if } n \neq n^{\prime}\right), \quad m\left(I \backslash \bigsqcup_{n \in \mathbb{N}} J_{n}\right)=0,
$$

where $m$ is the Lebesgue measure. Put $\hat{J}_{n}:=\bigsqcup_{k=1}^{n} J_{k}$. Since $\hat{J}_{n} \in \mathcal{O}_{1}$ holds, then $\left(\mathbb{R}^{3}, d_{\hat{J}_{n}}, 0\right) \in \mathcal{T}\left(X, g_{\Lambda}\right)$. If we put $\Phi_{J}(\zeta):=\int_{x \in J} \frac{d x}{\left|\zeta-\left(x^{\alpha}, 0,0\right)\right|}$, then we can see

$$
\left|\Phi_{\hat{J}_{n}}(\zeta)-\Phi_{I}(\zeta)\right| \leq \frac{m\left(I \backslash \hat{J}_{n}\right)}{D} \rightarrow 0 \quad(\text { as } n \rightarrow \infty)
$$

and we can take the constants in (A3-6) independent of $n$ by using Proposition 6.2. Therefore, we obtain $\left\{\left(\mathbb{R}^{3}, d_{\hat{J}_{n}}, 0\right)\right\}_{n} \stackrel{\mathrm{GH}}{\longrightarrow}\left(\mathbb{R}^{3}, d_{I}, 0\right)$. 
Finally, let $I \in \mathcal{B}_{+}\left(\mathbb{R}^{+}\right)$. Since the Lebesgue measure is the Radon measure, there exist $U_{n} \subset \mathcal{O}_{1}$ for any $n$ such that $I \subset U$ and $m(U) \leq m(I)+\frac{1}{n}$. Then we have $\left|\Phi_{I}(\zeta)-\Phi_{U_{n}}(\zeta)\right| \leq \frac{1}{n D}$, we have $\left\{\left(\mathbb{R}^{3}, d_{U_{n}}, 0\right)\right\}_{n} \stackrel{\mathrm{GH}}{\longrightarrow}\left(\mathbb{R}^{3}, d_{I}, 0\right)$ by the similar argument. Here, the positivity of $m(I)$ is necessary since $C_{0}$ in (A5) is given by $\int_{I} \frac{d x}{1+x^{\alpha}}>0$ by (4).

By Theorem 8.17, we can see $\left(\mathbb{R}^{3}, h_{0}, 0\right)$ and $\left(\mathbb{R}^{3}, \frac{1}{|\zeta|} h_{0}, 0\right)$ are also contained in $\mathcal{B}_{+}\left(\mathbb{R}^{+}\right)$. The author does not know whether any other metric spaces may appear as the tangent cone at infinity of $\left(X, g_{\Lambda}\right)$ or not.

\section{On the geometry of the limit spaces}

In this section, we study the geometry of $\left(\mathbb{R}^{3}, d_{0}^{\infty}\right)$, and conclude that there are no isometry between $\left(\mathbb{R}^{3}, d_{0}^{\infty}\right)$ and $\left(\mathbb{R}^{3}, h_{0}\right)$, and between $\left(\mathbb{R}^{3}, d_{0}^{\infty}\right)$ and $\left(\mathbb{R}^{3}, \frac{1}{|\zeta|} h_{0}\right)$

Proposition 9.1. $\left(\mathbb{R}^{3}, \frac{1}{|\zeta|} h_{0}\right)$ is the Riemannian cone $S^{2} \times \mathbb{R}^{+}$, where the Riemannian metric on $S^{2}$ is the homogeneous one whose area is equal to $\pi$.

Proof. Put $\zeta=\left(\zeta_{1}, \zeta_{2}, \zeta_{3}\right) \neq 0$ and $r=\sqrt{\zeta_{1}^{2}+\zeta_{2}^{2}+\zeta_{3}^{2}}$, and let $g_{S^{2}}$ be the standard Riemannian metric on $S^{2}$ with constant curvature and volume $4 \pi$. Then by putting $R:=2 \sqrt{r}$, we have

$$
\frac{1}{|\zeta|} h_{0}=\frac{1}{r}\left((d r)^{2}+r^{2} g_{S^{2}}\right)=(d R)^{2}+R^{2} \cdot \frac{g_{S^{2}}}{4} .
$$

Next we review the notion of polar spaces, introduced by Cheeger and Colding in [5] then show that the metric space $\left(\mathbb{R}^{3}, d_{0}^{\infty}\right)$ never be a polar space.

Let $Y$ be a metric space, and suppose that there is a tangent cone $Y_{y}$ at $y \in Y$. Then we can consider tangent cones at any points in $Y_{y}$. The tangent cones obtained by repeating this process are called iterated tangent cones of $Y$. A point $x$ in a length space $X$ is called a pole if there is a ray $\gamma:[0, \infty) \rightarrow X$ and $t \geq 0$ for any $\underline{x} \neq x$ such that $\gamma(0)=x$ and $\gamma(t)=\underline{x}$. Here, the ray $\gamma:[0, \infty) \rightarrow X$ is a continuous curve such that the length of $\left.\gamma\right|_{\left[t_{0}, t_{1}\right]}$ is equal to $\left|\gamma\left(t_{0}\right) \gamma\left(t_{1}\right)\right|$.

Definition $9.2([5])$. The metric space $Y$ is called a polar space if all of the base points of the iterated tangent cones of $Y$ are poles. 
For example, let $C(X)$ be a metric cone of a metric space $X$. Then every $\gamma$ defined by $\gamma(t):=(x, t) \in X \times \mathbb{R}^{+}=C(X)$ is a ray, hence the base points of any metric cones are poles. Now, since $\left(\mathbb{R}^{3}, \frac{1}{|\zeta|} h_{0}\right)$ is a Riemannian cone of a smooth compact Riemannian manifold, then all of the iterated tangent cones are $\left(\mathbb{R}^{3}, \frac{1}{|\zeta|} h_{0}\right)$ itself or $\left(\mathbb{R}^{3}, h_{0}\right)$. Consequently, we can conclude that $\left(\mathbb{R}^{3}, \frac{1}{|\zeta|} h_{0}\right)$ is polar. Obviously, $\left(\mathbb{R}^{3}, h_{0}\right)$ is also polar. We can also see in the similar way that $\left(\mathbb{R}^{3},\left(1+\frac{\theta}{|\zeta|}\right) h_{0}\right)$ is polar. On the other hand we can show the next proposition.

Proposition 9.3. The origin $0 \in \mathbb{R}^{3}$ is not a pole of the metric space $\left(\mathbb{R}^{3}, d_{0}^{\infty}\right)$. In particular, $\left(\mathbb{R}^{3}, d_{0}^{\infty}\right)$ is neither a polar space nor a metric cone of any metric spaces.

Proof. First of all we show that $0 \in \mathbb{R}^{3}$ is not a pole with respect to $d_{0}^{\infty}$. Put $p:=(1,0,0) \in \mathbb{R}^{3}$, and suppose that there is a ray $\gamma:[0, \infty) \rightarrow \mathbb{R}^{3}$ such that $\gamma(0)=0$ and $\gamma\left(t_{0}\right)=p$ for some $t_{0}>0$. Then we have

$$
d_{0}^{\infty}\left(\gamma\left(s_{0}\right), \gamma\left(s_{1}\right)\right)=\int_{s_{0}}^{s_{1}} \sqrt{\Phi_{0}^{\infty}(\gamma(t))}\left|\gamma^{\prime}(t)\right| d t
$$

for any $0 \leq s_{0}<s_{1}$.

For $\delta>0$, let

$$
A_{\delta}:=\left\{t \in \mathbb{R} ;\left|\gamma_{\mathbb{C}}(t)\right| \geq \delta\right\} .
$$

Then there is a sufficiently small $\delta$ such that $A_{\delta} \cap\left(0, t_{0}\right) \neq \emptyset$ and $A_{\delta} \cap\left(t_{0}, \infty\right) \neq$ $\emptyset$. This is because the length of $\left.\gamma\right|_{I}$ becomes infinity for any small interval $I \subset \mathbb{R}$ if not. Since $A_{\delta}$ is closed and does not contain $t_{0}$, we can take a connected component $\left(a_{0}, a_{1}\right)$ of $\mathbb{R} \backslash A_{\delta}$ containing $t_{0}$. Then we can see that $\left|\gamma_{\mathbb{C}}\left(a_{0}\right)\right|=\left|\gamma_{\mathbb{C}}\left(a_{1}\right)\right|=\delta$ and $\left|\gamma_{\mathbb{C}}(t)\right|<\delta$ for any $t \in\left(a_{0}, a_{1}\right)$. Now define $\tilde{\gamma}:\left[0, a_{1}\right] \rightarrow X$ by

$$
\tilde{\gamma}(t):=\left\{\begin{array}{cc}
\left(\gamma_{\mathbb{R}}(t), e^{i \theta} \gamma_{\mathbb{C}}(t)\right) & \left(0 \leq t \leq a_{0}\right) \\
e^{i \theta} P_{\left.\gamma\right|_{\left[a_{0}, a_{1}\right]}}(t) & \left(a_{0} \leq t \leq a_{1}\right)
\end{array}\right.
$$

where $\theta$ is defined by $e^{i \theta} \gamma_{\mathbb{C}}\left(a_{0}\right)=\gamma_{\mathbb{C}}\left(a_{1}\right)$. Recall that $P_{\gamma_{\left[a_{0}, a_{1}\right]}}$ is already defined in Lemma 7.4. Then by applying Lemma 7.4, we can see that the length of $\tilde{\gamma}$ is strictly less than the length of $\left.\gamma\right|_{\left[0, a_{1}\right]}$, therefore $\gamma$ is not the ray, which is the contradiction. Hence $0 \in \mathbb{R}^{3}$ is not the pole.

Now we can check that the $\mathbb{R}^{+}$-action on $\mathbb{R}^{3}$ defined by the scalar multiplication is homothetic with respect to $d_{0}^{\infty}$, then the tangent cone of $\left(\mathbb{R}^{3}, d_{0}^{\infty}\right)$ at 0 is itself. Consequently, $\left(\mathbb{R}^{3}, d_{0}^{\infty}\right)$ is not a polar space.

Suppose that $\left(\mathbb{R}^{3}, d_{0}^{\infty}\right)$ is the metric cone of some metric spaces $X$, then the origin 0 is nothing but the base point of the metric cone. Since the base point of the metric cone is always a pole, hence we have the contradiction. 
Now we obtain the next corollary.

Corollary 9.4. There is no isometry between $\left(\mathbb{R}^{3}, d_{0}^{\infty}\right)$ and $\left(\mathbb{R}^{3}, h_{0}\right)$, and between $\left(\mathbb{R}^{3}, d_{0}^{\infty}\right)$ and $\left(\mathbb{R}^{3}, \frac{1}{|\zeta|} h_{0}\right)$.

\section{References}

[1] Michael T. Anderson, Peter B. Kronheimer, and Claude LeBrun. Complete Ricci-flat Kähler manifolds of infinite topological type. Comm. Math. Phys., 125(4):637-642, 1989.

[2] V. N. Berestovskilu. "Submetries" of three-dimensional forms of nonnegative curvature. Sibirsk. Mat. Zh., 28(4):44-56, 224, 1987.

[3] V. N. Berestovskii and Luis Guijarro. A metric characterization of Riemannian submersions. Ann. Global Anal. Geom., 18(6):577-588, 2000.

[4] Jeff Cheeger and Tobias H. Colding. Lower bounds on Ricci curvature and the almost rigidity of warped products. Ann. of Math. (2), 144(1):189-237, 1996.

[5] Jeff Cheeger and Tobias H. Colding. On the structure of spaces with Ricci curvature bounded below. I. J. Differential Geom., 46(3):406-480, 1997.

[6] Tobias Holck Colding and William P. Minicozzi, II. On uniqueness of tangent cones for Einstein manifolds. Invent. Math., 196(3):515-588, 2014 .

[7] Tobias Holck Colding and Aaron Naber. Characterization of tangent cones of noncollapsed limits with lower Ricci bounds and applications. Geom. Funct. Anal., 23(1):134-148, 2013.

[8] R. Goto. On hyper-Kähler manifolds of type $A_{\infty}$. Geom. Funct. Anal., 4(4):424-454, 1994.

[9] Mikhael Gromov. Structures métriques pour les variétés riemanniennes, volume 1 of Textes Mathématiques [Mathematical Texts]. CEDIC, Paris, 1981. Edited by J. Lafontaine and P. Pansu.

[10] Misha Gromov. Metric structures for Riemannian and non-Riemannian spaces. Modern Birkhäuser Classics. Birkhäuser Boston, Inc., Boston, MA, english edition, 2007. Based on the 1981 French original, With appendices by M. Katz, P. Pansu and S. Semmes, Translated from the French by Sean Michael Bates.

[11] Kota Hattori. The volume growth of hyper-Kähler manifolds of type $A_{\infty}$. J. Geom. Anal., 21(4):920-949, 2011. 
[12] G. Perelman. A complete Riemannian manifold of positive Ricci curvature with Euclidean volume growth and nonunique asymptotic cone. In Comparison geometry (Berkeley, CA, 1993-94), volume 30 of Math. Sci. Res. Inst. Publ., pages 165-166. Cambridge Univ. Press, Cambridge, 1997. 